\title{
Domains and domain walls in multiferroics
}

Donald M. Evans ${ }^{1}$, Vincent Garcia ${ }^{2, *}$, Dennis Meier ${ }^{1}$, and Manuel Bibes ${ }^{2}$

\begin{abstract}
Multiferroics are materials combining several ferroic orders such as ferroelectricity, ferro- (or antiferro-) magnetism, ferroelasticity and ferrotoroidicity [1]. They are of interest both from a fundamental perspective, as they have multiple (coupled) non-linear functional responses - providing a veritable myriad of correlated phenomena, and because of the opportunity to apply these functionalities for new device applications. One application is, for instance, in no-volatile memory, which has led to special attention being devoted to ferroelectric and magnetic multiferroics. For this application, the vision is to combine the low writing power of ferroelectric information with the easy, non-volatile, reading of magnetic information to give a 'best of both worlds' computer memory: however, for this to be realised the two ferroic orders need to be intimately linked via the magnetoelectric effect. The magnetoelectric coupling - the way polarization and magnetization reverse - is manifested by the formation and interactions of domains and domain walls, and so to understand how to engineer future devices one must first understand the interactions of domains and domain walls. In this article, we provide a short introduction to the domain formation in ferroelectrics and ferromagnets, as well as different microscopy techniques that enable the visualization of such domains. We then review the recent research on multiferroic domains and domain walls, including their manipulation and intriguing properties, such as enhanced conductivity and anomalous magnetic order. Finally, we discuss future perspectives concerning the field of multiferroic domain walls and emergent topological structures such as ferroelectric vortices and skyrmions.
\end{abstract}

Keywords: multiferroic, domains, domain walls, microscopy

\section{Domain structures in (multi-)ferroics}

\subsection{Introduction to ferroic domains and domain walls}

Ferroic materials are defined by the appearance of an order parameter (e.g., elastic, electric or magnetic) at a nondisruptive phase transition. This order parameter can point in at least two symmetrically equivalent directions (polarities) between which it can be switched by the application of an external field. When cooling through the phase transition in zero-field, the polarities have the same energy and, as a consequence, both polarities appear inside the ferroic material. These regions are called domains and the interfaces that separate them are call domain walls. While this shows, trivially, that multiple domains form naturally in ferroics, the details of how many domains, their size, and where they form, depend on several energy terms, as well as the local defect structure. In the following, we will give a brief outline of how ferroic domains and domain walls form, using a ferroelectric as an illustrative example; although, as we will see, analogous arguments can be made for the formation of domains and domains walls in ferromagnets. For a more complete and in-depth descriptions of the physics of ferroelectric and ferromagnetic domains we refer to, for instance, the textbooks by Tagantsev et al. [2] and by Hubert \& Schäfer [3].

A proper ferroelectric is a material for which the spontaneous electric polarization plays the role of the primary symmetry breaking order parameter, which can completely describe the phase transition into the ferroic state [4],[5]. Practically, this means energy contributions related to this order parameter are very important in the system, particularly for the domain formation. For instance, in ferroelectrics, surfaces perpendicular to the ferroelectric polarization comprise bound charges that create a strong depolarizing field (Fig. la): this is a major driving force for domain formation. The depolarizing field can be minimized by either (i) screening the surface charges of the ferroelectric with surface adsorbates or metallic electrodes (Fig. 1b), or (ii) the formation of ferroelectric domains, for example, $180^{\circ}$ domains, so that the net polarization at the surface averages to zero (Fig. 1c). The number and size of the ferroelectric domains that form will

${ }^{1}$ Department of Materials Science and Engineering, Norwegian University of Science and Technology (NTNU), Trondheim, Norway

${ }^{2}$ Unité Mixte de Physique CNRS, Thales, Univ. Paris Sud, Université Paris-Saclay, 91767 Palaiseau, France

*Email : vincent.garcia@cnrs-thales.fr 
depend on the details of the boundary conditions (Fig. 1d), such as crystal size, shape, orientation, local defect structure and, critically, the energy costs associated with the insertion of domain walls. Thus, depending on the boundary conditions, a thin ferroelectric film can either form a single-domain state or break into multiple nanodomains [6], which has been used to engineer ferroelectric domains [7]-[10]. This can easily be observed by half covering a large single crystal with electrodes and cooling the sample through its Curie temperature: the area under the electrodes will form larger domains than the uncovered area due to the presence of screening charges from the electrodes, while the area in air will have a finer domain structure due to the depolarising fields (see, e.g., Gilletta 1972 [11]). Importantly, from these simplistic arguments, it can be seen that the formation of domains and domain walls is a natural property of any ferroic crystal. Note that this applies in the case of zero applied electric field; applying an electric field will affect both the transition temperature and the domain formation, see e.g. Merz 1953 [12].

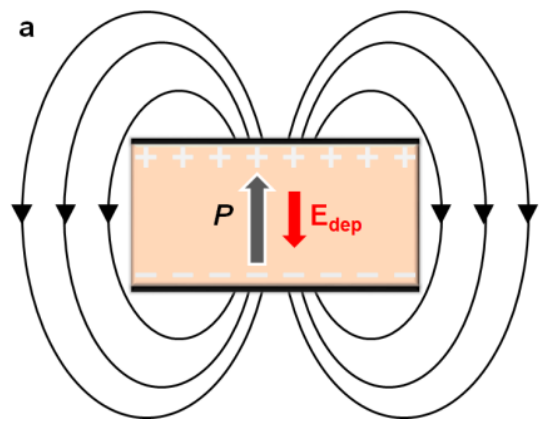

b

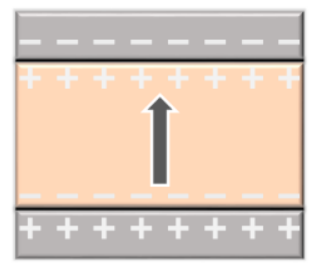

C

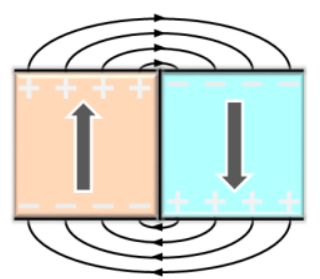

d

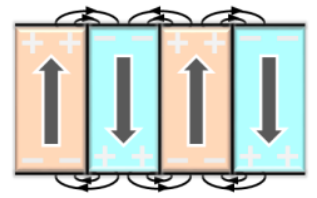

Fig. 1: Domain formation in ferroelectrics. (a) Black field lines represent the stray field from a ferroelectric monodomain state. The grey arrow shows the polarisation direction, $\boldsymbol{P}$. The build-up of stray electric fields induces a field in the opposite direction, the depolarising field, $\boldsymbol{E}_{\mathrm{dep}}$, indicated by the red arrow. (b) Complete screening of the ferroelectric polarisation by surface charges. (c) $180^{\circ}$ domain wall leading to a smaller electric stray field that reduces the internal depolarising field. (d) Representative multidomain state showing the much smaller electric stray fields. Note that the number of domains formed will depend on several energy terms including the energy cost of having a domain wall, the size of the depolarising field, and the presence of uncompensated surface charges.

In general, the size of the domains that form can be predicted by what is now known as Kittel's scaling law [13], [14]. Although Kittel attributes the original idea of size effects of domains to Frenkel and Doefman [15], it was Kittel's work on ferromagnets that provided the scaling relationship that now bears his name - the domain width is inversely proportional to one over the square root of their thickness as detailed below. Originally, his mathematical formalism considered only three contributions to the free energy as relevant, that is, the surface energy of a domain wall, the magnetic field energy of the configuration, and the anisotropy of the spin orientation. Kittel's work on ferromagnets was then expanded to ferroelectrics by Mitsui and Furuichi in 1952 [16], showing that the fundamental scaling remains the same. It has also now been experimentally verified in ferroelectrics and multiferroics down to tens of nanometre sample thicknesses [17], [18]. Some corrections to Kittel's law have been proposed by Scott [19] due to the finite size of domain walls, being particularly pertinent in ferroelectrics, where domain walls are usually about one order of magnitude thinner than in ferromagnets.

To illustrate Kittel's scaling law, we consider the simple case of open boundary conditions without surface screening in a film with $180^{\circ}$ striped-domains as illustrated in Fig. 1d. Here, the size of domains is determined by the competition between the energy of the domains and that of domain walls. The energy density, $E$, of the domains depends on their width, $\omega$, as $E=U \omega$, where $U$ is the volume energy density. The energy density of the domain walls depends on their number and so will be inversely proportional to $\omega$ as $E=\sigma d / \omega$, where $\sigma$ is the energy density per unit area of the wall and $d$ the thickness of the film. Minimizing the total energy gives rise to a square-root dependence of the domain size with the film thickness as $\omega=\sqrt{\sigma / U \times d}$ [14], known as Kittel`s law [14].

In the original form proposed for ferromagnets, it is the competition between magnetic exchange energy and demagnetizing field contributions that drives the domain formation: The magnetic exchange energy is minimized in singledomain states while the competing demagnetising field favours the formation of multi-domain states. Furthermore, additional factors arise from the size and shape of the magnetic domains and domain walls, which will depend on the magnetocrystalline and magnetostrictive energies of the system. While the magnetic exchange energy favours wide walls, so that neighbouring magnetic moments are almost parallel to each other, magnetocrystalline anisotropy promotes narrow walls so that almost all the magnetization of the system is aligned with the easy magnetic axis. As a result, the 
thickness of these magnetic domain walls depends strongly on material parameters, but typical values are in the order of a few tens of nanometers and up to hundreds of nanometers [20]. In contrast to ferromagnets, where the exchange energy is much larger than the magnetocrystalline anisotropy, in ferroelectrics the anisotropy and dipole-dipole interactions (the equivalent of exchange in these materials) are of the same order of magnitude [21]. As a consequence, ferroelectrics usually develop much thinner walls than ferromagnets, with typical thicknesses in the order of a few unit cells (see, for instance, Refs. [17], [22]-[26]). The smaller width of ferroelectric domain walls compared to magnetic domain walls implies that the order parameter changes orientation much faster.
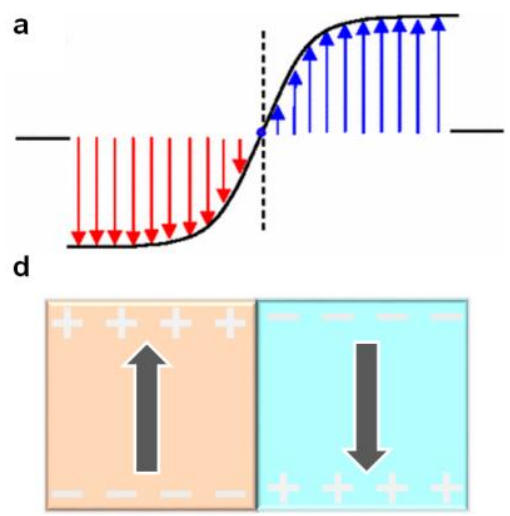

b
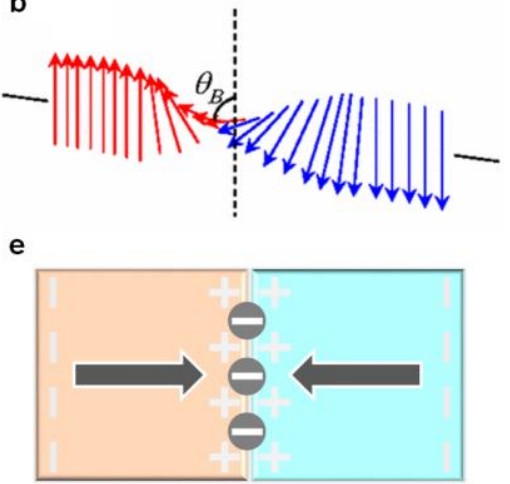

C
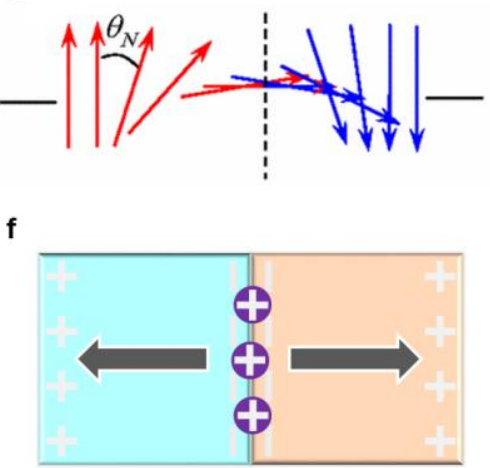

Fig. 2: Fundamental types of ferroic domain wall. Arrows represent the orientation of the order parameter (e.g., polarisation or magnetisation). (a) Ising-type wall: the polarisation/magnetisation does not rotate but decreases in magnitude through the wall. (b) Bloch-type wall: the polarisation/magnetisation does not change magnitude but rotates in the plane of the domain wall. (c) Néel-type wall: the polarisation/magnetisation does not change magnitude but rotates perpendicular to the plane of the domain wall. (a)-(c) are reprinted figure with permission from [27]. Copyright 2009 by the American Physical Society. (d) - (f) Charge states at ferroelectric $180^{\circ}$ walls. (d) Neutral $180^{\circ}$ domain wall. Positive and negative bound charges are denoted by symbols + and -, respectively (e) Head-to-head domain wall, where the polar discontinuity associated with the positive ferroelectric bound charges attract mobile negative charge carriers (grey). (f) Tail-to-tail domain wall, where the polar discontinuity associated with the negative ferroelectric bound charges attract mobile positive charge carriers (purple).

At the domain wall itself there are, conceptually, three different ways for the polar order to change, referred to as Ising-, Bloch-, and Néel-type walls. The three domain wall types are schematically illustrated in Fig. 2a-c. In Ising-type walls (Fig. 2a), the axis along which the order parameter points is fixed. Across the wall, only the magnitude of the polar order changes smoothly from up to down, going through zero at the center of the wall. These walls are generally the thinnest of order (sub)nanometer wide [22], [23]. Traditionally, it was assumed that $180^{\circ}$ domain walls in ferroelectrics are Ising-type walls. Recent experimental and theoretical work, however, has revealed domain walls with more complex structures (see, e.g., Refs. [27]-[30]). For ferroelectric Ising-type walls, as already mentioned above, both the anisotropy and dipole-dipole interactions are usually strong, and the energy difference between the paraelectric and ferroelectric phases is relatively small. Consequently, an abrupt change of polarization through the wall can readily be accommodated by a reduction of the magnitude of polarization as presented in Fig. 2a.

In the Bloch-type wall, the order parameter does not change size but rotates within the plane of the wall, so that at the center the corresponding vector is parallel to the plane of the domain wall (Fig. 2b). The Néel-type wall is characterized by a rotation of the order parameter perpendicular to the domain wall and at the wall center the respective vector lies orthogonal to the plane of the domain wall (Fig. 2c). Bloch- and Néel-type walls are most common in ferromagnets, where the energy difference between paramagnetic and ferromagnetic phases is large and, hence, the norm of magnetic moments does not vary significantly [3]. These domain walls can be quite wide and extend up to hundreds of nanometers in width [20]. The three extreme cases shown in Fig. 2a to 2c are normally combined to describe domain walls in real systems, leading to mixed domain wall states. As already mentioned, it is important to note that Bloch- and Néel-type walls are not restricted to ferromagnets and, recently, there is a growing interest in non-Ising-type domain walls in ferroelectrics and the emergence of walls of Néel- and Bloch-type has been reported for different ferroelectric materials [31] such as $\mathrm{BaTiO}_{3}[32]$ and $\mathrm{Pb}(\mathrm{Zr}, \mathrm{Ti}) \mathrm{O}_{3}$ (PZT) [33]-[35]. Note that the illustrations in Fig. 2 are for domain walls at 
which the order parameter orientation changes by $180^{\circ}$; the details change for other situations, e.g., for $90^{\circ}$ walls, but the salient points remain true.

In multiferroics, the situation becomes even more interesting as electric and magnetic domains and domain walls coexist [36]-[39], giving rise to unusual magnetoelectric correlation phenomena as we discuss in section 2 and 3 (see also DOI:10.1515/PSR.2019.0014 and DOI:10.1515/PSR.2019.0016). Because of the coexistence of different types of ferroic order in multiferroics, it becomes important to consider whether the polarization or magnetization plays the role of the symmetry breaking order parameter and, thus, governs the formation of domains and domain walls. In this context, one typically distinguishes between proper and improper systems or, more precisely, between systems in which the ferroic order arises across a proper or improper phase transition [4], [5], [40]. For example, as described above, a ferroelectric phase transition is referred to as proper, if the spontaneous polarization is the primary symmetry breaking order parameter that drives the transition. This is the case in textbook ferroelectrics such as $\mathrm{BaTiO}_{3}, \mathrm{PZT}$, and $\mathrm{LiNbO}_{3}[2]$, [41]. Alternatively, electric order can arise as a secondary effect driven by coupling to, e.g., a structural [42]-[45] or magnetic order parameter [46]-[48]. The latter scenario, referred to as improper ferroelectricity (or pseudo-proper; see, e.g., [4], [40] for details), is realized in many multiferroics. For instance, in well-known model systems such as the hexagonal manganites, $R \mathrm{MnO}_{3}(R=\mathrm{Sc}, \mathrm{Y}$, In, Dy to Lu), which are reviewed in DOI:10.1515/PSR.2019.0014, and orthorhombic $\mathrm{TbMnO}_{3}$ and $\mathrm{DyMnO}_{3}$ with fascinating consequences for the domain walls [39], [49]-[52]. Here, due to the secondary nature of the spontaneous polarization, exotic charged domain walls arise spontaneously - a situation that is usually avoided due to the high electrostatic energy costs.

Assuming an Ising-type wall, a domain wall is said to be fully charged if the polarisation of neighbouring domains comes together head-to-head or tail-to-tail as shown in Fig. 2e and 2f, respectively [53]. The associated discontinuity in polarization $\boldsymbol{P}$ leads to uncompensated domain wall bound charges (div $\boldsymbol{P} \neq 0$ ), which require screening. As a consequence, mobile carriers redistribute, promoting anomalous electronic transport properties at head-to-head and tail-to-tail walls, including highly conducting and insulating states [53]-[58], as well as the formation of electronic inversion layers [59]. Charged domain walls thus represent a natural type of two-dimensional system with inherent functional properties, which can be injected, moved and erased on demand [60]-[64]. Additional functionalities arise at domain walls with a strong coupling between electric and magnetic degrees of freedom, enabling magnetic field control of the local electronic charge states as discussed in section 3 [36], [39], [65]. For a more comprehensive or more technical coverage of charged domain walls in ferroelectrics and multiferroics, we refer to recent reviews [66]-[69], and the specific studies highlighted in the following sections.

\subsection{Visualization of domains}

The rapid progress that has been made in the understanding of magnetic and electric domains relies on the recent developments in microscopy techniques with high sensitivity and unprecedented spatial resolution. Nowadays, even atomic level resolution is readily available with transmission electron microscopy (TEM) (see, e.g., DOI:10.1515 /PSR.2019.0068 and references therein (ref. [70]). In this review, we discuss different microscopy methods that allow for studying the formation and interaction of ferroic domains in spatially resolved measurements on nano- to microscopic length scales. In multiferroics, one specific difficulty arises for domain imaging as one must distinguish contributions from at least two coexisting types of ferroic order. Many available techniques are simultaneously sensitive to magnetism and ferroelectricity, making the distinction between the two signals challenging. For instance, photoemission electron microscopy (PEEM) based on X-ray linear dichroism (XLD) is sensitive to the asymmetry of the electronic charge distribution and, hence, to contributions from both ferroelectric and antiferromagnetic domains. Another example is scanning probe microscopy (SPM); the magnetic tip used for magnetic force magnetometry (MFM), for example, will probe contributions from the stray field of the magnetic domains as well as the electrostatic fields from the ferroelectric domains and surface charges (as in electrostatic force magnetometry (EFM)). In the following, we give examples of different techniques with which magnetic and ferroelectric domains can be observed independently as illustrated based on measurements taken on both intrinsic and artificial multiferroics (Fig. 3).

\subsubsection{Optical microscopy}

In bulk single crystals, domains may reach sizes in the micron range compatible with optical microscopy. In this case, ferroelectric domains can be imaged with the birefringent contrast [71]-[73], or selective etching [74], while magnetic domains are observed via the magneto-optical Kerr effect. Figure 3a,b illustrates this on an artificial multiferroic system based on a thin ferromagnetic film $\left(15 \mathrm{~nm}\right.$ of $\left.\mathrm{Co}_{0.6} \mathrm{Fe}_{0.4}\right)$ deposited on a ferroelectric single crystal $\left(\mathrm{BaTiO}_{3}\right)(\mathrm{Ref}$ 
[75]). The ferroelectric domains of $\mathrm{BaTiO}_{3}$ organize in the form of stripes with $90^{\circ}$ ferroelastic domain walls (a1-a2 domains corresponding to in-plane polarization in Fig. 3a). Strain coupling between the $\mathrm{Co}_{0.6} \mathrm{Fe}_{0.4}$ thin film and the underlying $\mathrm{BaTiO}_{3}$ single crystal induces uniaxial magnetoelastic anisotropy with orthogonal easy axes between neighbouring domains, resulting in an imprinted stripe pattern (Fig. 3b).

\subsubsection{Scanning probe microscopy}

SPM allows nanoscale investigation of both ferroelectric domains, via piezoresponse force microscopy (PFM), and ferromagnetic domains, via MFM. In MFM, the magnetic tip experiences an attracting or repelling force depending on its relative magnetization orientation compared to that of the sample. This force induces a phase lag on the oscillation of the tip and MFM images show the spatial distribution of the magnetic stray field coming from the sample.
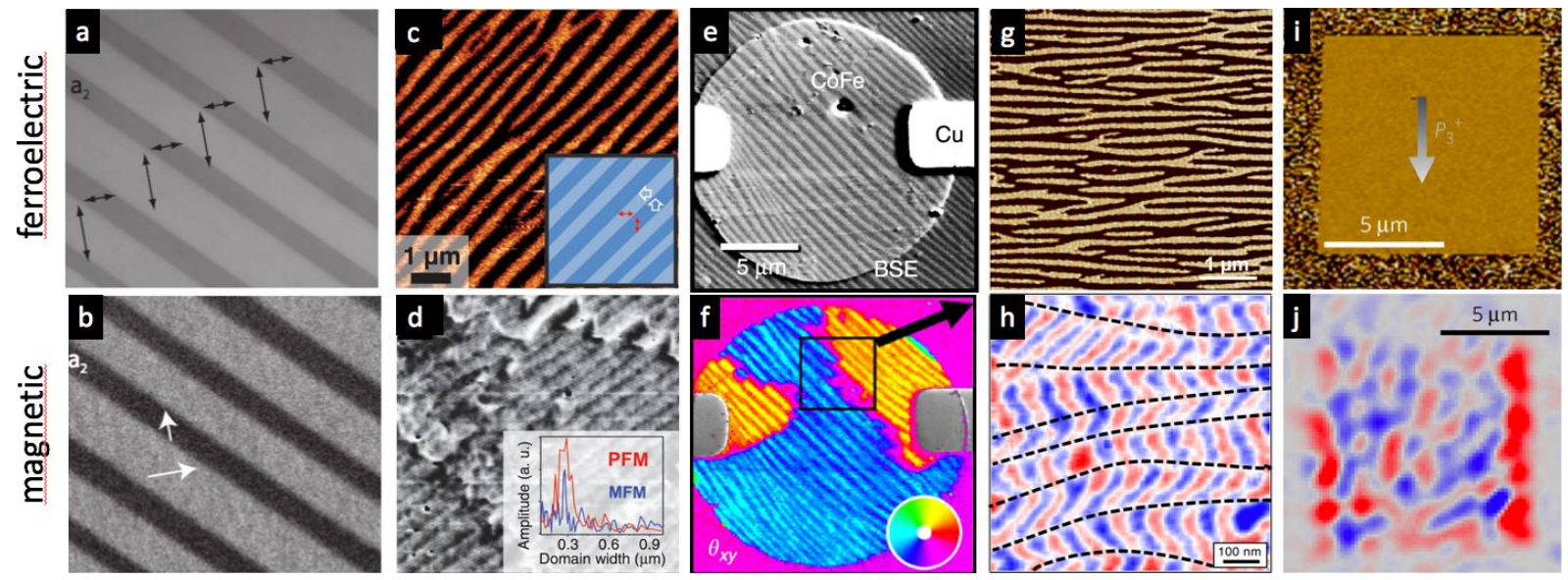

Fig. 3: Imaging magnetic and ferroelectric domains in artificial and intrinsic multiferroics. (a) Polarized optical microscopy image of (a) the al-a2 birefringent ferroelectric domains of a $\mathrm{BaTiO}_{3}$ single crystal and (b) the magnetic domains of the uniaxial $\mathrm{Co}_{0.6} \mathrm{Fe}_{0.4}$ layer $\left(15 \mathrm{~nm}\right.$ ) grown on top. (a-b are from reference [75]). (c) $\mathrm{PFM}$ image of a $\mathrm{BiFeO}_{3}$ film grown on $\mathrm{DySCO}_{3}(110)$ substrate and (d) MFM image on the same sample after the growth of a Pt/Co $\mathrm{Co}_{0.9} \mathrm{Fe}_{0.1}$ bilayer on top under a magnetic field of $20 \mathrm{mT}$. In the inset, the Fourier analysis indicates the correlation between the PFM and MFM patterns. (c-d are reprinted figure with permission from [76]. Copyright 2013 by the American Physical Society). (e) Ferroelectric domain structure of $\mathrm{BiFeO}_{3}$ imaged using the back-scattered electron (BSE) intensity with a $\mathrm{Co}_{0.9} \mathrm{Fe}_{0.1}$ thin-film circular disk and $\mathrm{Cu}$ electrode on the side. (f) Simultaneously acquired SEMPA image of the magnetic structure. The magnetization direction is represented by colors as indicated by the color wheel. (e-f reprinted by permission from Springer Nature, taken from [77]. Copyright 2015 by Springer Nature.) (g) In-plane PFM image of the stripeddomain structure of a 30-nm-thick $\mathrm{BiFeO}_{3}$ thin film grown on $\mathrm{DyScO}_{3}(110)$ and (h) NV-magnetometry image of the stray field produced by the spin cycloid of the same film. (g-h reprinted by permission from Springer Nature, taken from [78]. Copyright 2017 by Springer Nature.) (i) In-plane PFM image of a single ferroelectric domain written with the trailing field of the SPM tip on a $\mathrm{BiFeO}_{3}$ thin film grown on a $\mathrm{SrTiO}_{3}(001)$ substrate. (j) Reconstructed SHG image showing two types of submicron antiferromagnetic domains in the ferroelectric domain showed in (i). (i)-(j) reprinted by permission from Springer Nature, taken from [79]. Copyright 2017 by Springer Nature.

The probe tips used for MFM are usually coated with ferromagnetic materials and their metallicity enables their subsequent use for PFM. PFM uses the fact that all ferroelectric materials are piezoelectric [80]. By applying an alternating voltage between the SPM tip and the ferroelectric in contact, the ferroelectric will vibrate at the same frequency as the voltage excitation. The phase shift of the response is directly connected to the out-of-plane polarization orientation of the ferroelectric. In the same manner, the torsion of the tip enables information of the in-plane components of the polarization. An example is given in Fig. 3, where the MFM contrast coming from a 2.5-nm-thick $\mathrm{Co}_{0.9} \mathrm{Fe}_{0.1}$ amorphous layer (Fig. 3d) is correlated to the PFM contrast from the ferroelectric domain pattern (Fig. 3c) in the underlying layer of $\mathrm{BiFeO}_{3}$ (Ref. [76]). In this configuration, the surface bound charges of $\mathrm{BiFeO}_{3}$ are screened by the magnetic top electrode and the MFM signal is "pure". This correlation attests for the coupling between the ferromagnetic domains in $\mathrm{Co}_{0.9} \mathrm{Fe}_{0.1}$ and the antiferromagnetic domains in $\mathrm{BiFeO}_{3}$, the latter being magnetoelectrically coupled with the ferroelectric order.

In order to directly image the stray field coming from the antiferromagnetic domains in multiferroics such as $\mathrm{BiFeO}_{3}$ (see DOI:10.1515/PSR.2019.0070 for details on $\mathrm{BiFeO}_{3}$ ), a scanning probe technique with higher sensitivity than MFM 
is required. Recently, scanning nitrogen-vacancy (NV) magnetometry was developed in which stray fields down to a few $\mu \mathrm{T}$ can be detected [81]. In bulk [82] and lightly strained films of the antiferromagnetic $\mathrm{BiFeO}_{3}$ [83], the magnetoelectric interaction stabilizes the formation of a spin cycloid - originally probed by Sosnowska et al. using neutron diffraction [84] - whose propagation vector is coupled to the polarization direction. Gross et al. were able to use NV magnetometry to visualize the stray field emanating from this 70-nm-long spin cycloid (Fig. 3h) in striped domain patterns of $\mathrm{BiFeO}_{3}$ thin films [78] (Fig. 3g). The in-plane compressive strain imposed by the $\mathrm{DyScO}_{3}$ substrate lifts the degeneracy between the three possible propagation vectors of spin cycloid for each ferroelectric domain, resulting in the in-plane $90^{\circ}$ rotation of the magnetic signal between alternating ferroelectric domains (Fig. 3h).

\subsubsection{Scanning electron microscopy}

In a scanning electron microscope with polarization analysis (SEMPA), the low energy secondary electrons are spin-polarized and give information on the magnetization orientation of the ferromagnetic structure, while the high energy elastically back-scattered electrons (BSE) are sensitive to the crystal structure and lattice distortions to reveal the polarization orientation of the ferroelectric structure. Combining SEMPA and BSE, Zhou et al. were able to simultaneously image the local in-plane vector magnetization of the $\mathrm{Co}_{0.9} \mathrm{Fe}_{0.1}$ layer (Fig. 3f) grown over the striped-domain ferroelectric structure of $\mathrm{BiFeO}_{3}$ (Fig. 3e) depicted previously [77]. The comparison of the SEMPA and BSE images shows the close correlation between the magnetic structure of the $\mathrm{Co}_{0.9} \mathrm{Fe}_{0.1}$ film and the striped ferroelectric domain structure of the underlying $\mathrm{BiFeO}_{3}$ layer. Within a stripe, which is about $250 \mathrm{~nm}$ wide, the magnetization is aligned parallel or anti-parallel to the in-plane surface projection of the electric polarization.

\subsubsection{Photoemission electron microscopy}

A possible way to discriminate ferroelectric and magnetic domain contrasts is to take advantage of the potentially different Curie and Néel temperatures of the multiferroic. This approach was used by Zhao et al. [85] to distinguish the antiferromagnetic contribution and the ferroelectric one in PEEM images of $\mathrm{BiFeO}_{3}$ thin films. A careful comparison of the PEEM contrast under and above the Néel temperature of $\mathrm{BiFeO}_{3}\left(T_{\mathrm{N}}=640 \mathrm{~K}\right)$ allows to separate the "pure" ferroelectric contrast from the multiferroic one with a resolution down to $\approx 20 \mathrm{~nm}$. A smart combination of XMLD- (X-ray magnetic linear dichroism) and XMCD- (X-ray magnetic circular dichroism) PEEM imaging (mapping spin and orbital magnetic moments) is a very powerful tool.

\subsubsection{Second harmonic generation}

Second-harmonic generation (SHG), that is, the frequency doubling of light in a material, is a powerful technique to sense complex magnetic and electric structures [86]-[88]. It is well suited to probe insulators with a resolution limited by the wavelength of the light in the submicron range. In bulk multiferroics, SHG domain imaging was pioneered by Fiebig [86]. More recently, Chauleau et al. used SHG to probe antiferromagnetic domains across a single ferroelectric domain of a $\mathrm{BiFeO}_{3}$ thin film (Fig. 3i) [79]. They performed the experiments in transmission while varying the incident polarization of the light and analysed the full angular dependence of each pixel. The 110-nm-thick $\mathrm{BiFeO}_{3}$ thin film was epitaxially grown on $\mathrm{SrTiO}_{3}(001)$ at a compressive strain that destroys the cycloidal spin structure and stabilizes a slightly-canted antiferromagnetic state arising from the Dzyaloshinskii-Moriya interaction [83]. In this G-type antiferromagnetic system, the $\mathrm{Fe}^{3+}$ spins are aligned along three possible antiferromagnetic vectors of the (111) plane, perpendicular to the ferroelectric polarization. As depicted in Fig. 3j, only two types of submicron antiferromagnetic domains were observed in this single $\mathrm{BiFeO}_{3}$ ferroelectric domain, suggesting that the magnetoelastic energy lifts the degeneracy between the three types of antiferromagnetic domains.

\subsubsection{Combined imaging experiments}

While the application of just one experimental method that can image both electric and magnetic domains is clearly intriguing, the majority of studies on multiferroics still makes use of two or more complementary techniques to image the coexisting domains and domain walls and cover all relevant length scales. The latter is nicely reflected looking at the research studies on multiferroics involving PFM. PFM by itself provides access to the distribution of ferroelectric domains [80]. The combination of PFM with PEEM and X-ray resonant magnetic scattering lead to the discovery of coupled ferroelectric and ferromagnetic domain in $\mathrm{BiFeO}_{3}$ thin films [85] and interfacial multiferroicity in $\mathrm{Fe} / \mathrm{BaTiO}_{3}$ 
and $\mathrm{Co} / \mathrm{BaTiO}_{3}$ heterostructures [89]. PFM in combination with $\mathrm{NV}$ magnetometry disclosed the coupling between ferroelectric and antiferromagnetic domain in $\mathrm{BiFeO}_{3}$ [78] (see also section 3). In addition, PFM has been combined with many other methods such as TEM, optical microscopy, as well as SHG in order to understand and disentangle the formation of electric and magnetic domains in different multiferroic materials. For a more extended review on PFM, see [80].

\section{Domain walls in multiferroics}

The recent discovery of functional electronic and magnetic properties at multiferroic domain walls triggered worldwide attention and initiated a shift in the research focus away from domains and towards domain walls. The domain wall research is driven by the idea to develop a new generation of agile interfaces/2D systems that remain spatially mobile after a material has been synthesized and implemented into a device structure [60]. Going beyond just conducting domain walls, as observed in ferroelastic and ferroelectric materials, multiferroics offer additional degrees of freedom that arise from the magnetic order and the unusual couplings between their electric, magnetic and structural properties as discussed in the following.

\subsection{Domain wall types}

Across domain walls in multiferroics, one or more order parameters change from one direction to another and this can occur in many different ways depending on the type of involved ferroic orders, the direction of the order parameters in the adjacent domains (e.g. parallel $\left(180^{\circ}\right)$ or perpendicular $\left(90^{\circ}\right)$ to the wall), and the geometry and dimensions of the sample [90], [91]. The range of structures that arises within a domain wall in the presence of just one order parameter has already been addressed in section 1 (see Fig. 2). In order to classify the complex domain walls that occur in multiferroics with coexisting electric and magnetic order [24], [68], [92], a first distinction can be made based on the interaction of the respective domain walls as shown in Fig. 4a and 4b [67].

In multiferroics where electric and magnetic orders occur independently (type I), electric and magnetic domain walls can coincide, but they do not necessarily have to couple due to the different microscopic origin of the polarization $(P)$ and magnetization $(M)$ (Fig. 4a). This is different in systems where the magnetic order induces the spontaneous polarization (see also DOI:10.1515/PSR.2019.0016), or vice versa, so that both orders occur together (type II, Fig. 4b). In the latter case, every ferroelectric domain wall is also a magnetic domain wall [37], [93], representing a two-dimensional system with multiferroic properties different from the surrounding bulk. 
a

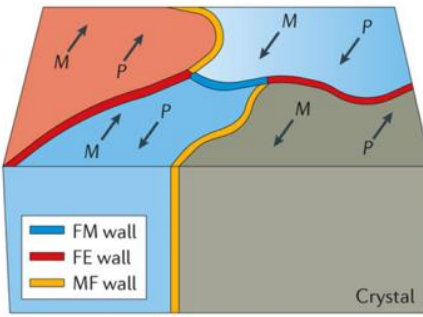

b

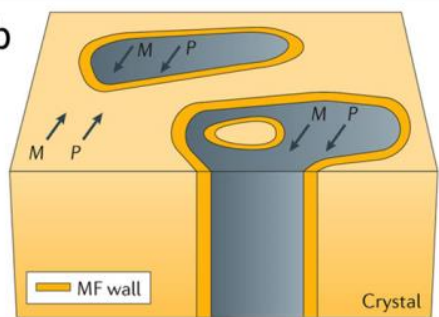

C
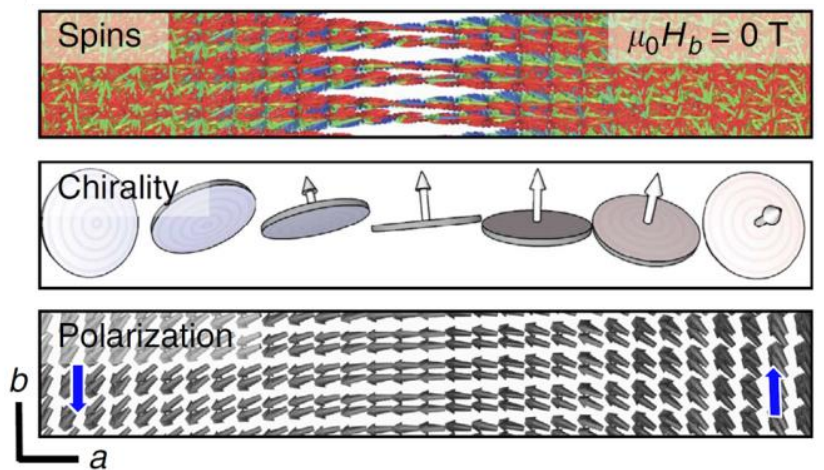

Fig. 4: Domain walls in multiferroics. (a) In type I multiferroics magnetic and electric order emerge independently and so do not need to coincide. This gives domain walls which are either magnetic (blue) or electric (red) in nature. If the domains happen to coincide then a multiferroic (orange) wall is formed, which points to a coupling which is not required by symmetry. (b) In type II multiferroics the magnetic order induces the electric order. Thus, ferroelectricity emerges at the magnetic phase transition. Due to this interdependence of order parameters, all ferroelectric domain walls are also magnetic domain walls and therefore multiferroic walls. (a) - (b) are reprinted by permission from Springer Nature, taken from [67]. Copyright 2016 by Springer Nature (c) Landau-Lifshitz-Gilbert simulation showing the complex evolution of spins (top), the spin chirality (middle), and electric polarisation (bottom) across a multiferroic domain wall in $\mathrm{Mn}_{0.95} \mathrm{Co}_{0.05} \mathrm{WO}_{4}$. Reprinted by permission from Springer Nature, taken from [36]. Copyright 2015 by Springer Nature.

A second, more advanced distinction is related to the conservation (or not) of the amplitude of the order parameter(s) across the wall. As introduced in section 1, we usually consider three fundamental types of ferroic domain walls, namely, Ising-, Néel-, and Bloch-type walls (Fig. 2). For Ising-type walls, upon crossing the wall the order parameter decreases, becomes zero at the centre and then increases again with the opposite polarity. For example, this is the situation for the ferroelectric walls in the type $\mathrm{I}_{\text {multiferroics }} \mathrm{BiFeO}_{3}$ and $\mathrm{ErMnO}_{3}$ [22], [94], where the polarization (and the associated structural distortion) is smaller at the core of the wall than in the domains [95]. In contrast, at the coexisting (anti)ferromagnetic domain walls, the amplitude of magnetic moments is usually conserved (non-Ising-type) and the order parameter rotates through the wall, potentially forming Bloch-, Néel-, and mixed Néel-Bloch-type walls (Fig. 2), or even more complex vortex-like walls states [96].

Particularly complex domain wall structures arise in type II multiferroics, where both the electric and magnetic

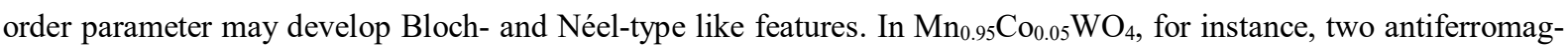
netic domain states of opposite chirality occur in the multiferroic state [36]. Across the domain walls, this change in chirality is realized via a continuous $180^{\circ}$ rotation of the material's easy-plane, forming a Bloch-type-like domain wall with respect to the spin-chirality vector $C=S_{\mathrm{i}} \times S_{\mathrm{j}}$ as illustrated in Fig. 4c. Interestingly, as $\mathrm{Mn}_{0.95} \mathrm{Co}_{0.05} \mathrm{WO}_{4}$ is an improper ferroelectric (section 1) with a magnetically induced polarization $\left(P \sim e_{\mathrm{ij}} \times\left(S_{\mathrm{i}} \times S_{\mathrm{j}}\right)\right.$ [47]), the electric order follows the rotation across the wall, which leads to Néel-type-like ferroelectric walls. Analogous to standard ferroelectric domain walls, such magnetically induced ferroelectric walls can carry a finite domain wall bound charge, which enables magnetic control of electronic domain wall states as we discuss in more detail in section 3 .

Remark about topology: The discontinuity of properties occurring at domain walls often leads the community to refer to them as topological defects, following concepts presented in the famous review by Mermin [93]. However, recent years have seen the emergence of topological spin textures (e.g. skyrmions) as an active field of research in nanomag netism and led to a stricter definition. Specifically, a given three-dimensional spin configuration is said to be topological if its topological charge $Q$ is an integer (or a half integer, as is the case of vortices and merons), and $Q$ is defined as $Q=$ $f q(x, y) d x d y$, where $q(x, y)=\frac{1}{1 \pi} n\left(\frac{d n}{d x} \times \frac{d n}{d n}\right)$ denotes the Pontryagin density. The topological charge is also-sometimes called winding number. In this definition, coplanar structures such as Ising, Bloch-and Néel type walls are not 
topological. For a more detailed discussion of topological objects in ferroies, and condensed matter systems in general, we refer the interested reader to Braun [94]

In summary, pronounced couplings between the electric and magnetic order parameters can occur in multiferroics, giving rise to a huge richness of domain wall structures and properties. Although the electric dipole and magnetic spin configurations at the atomic scale are rarely available - so that the exact wall types often remain unknown - it is clear that multiferroic domain walls can show unexpected and fascinating physical properties beyond the bulk properties. Today, we are only at the verge of discovering these exciting properties and novel findings and insight into the nanophysics of domain walls are to be expected in the near future, enabled by the continuous and ongoing progress in advanced microscopy techniques. Some of the recent key results in the field are reviewed in the following.

\subsection{Conduction in domain walls}

A little history - The emergence of conducting domain walls was postulated first in the 1960s and 70s based on macroscopic measurements in classic ferroelectrics like TGS (triglycine sulfate), $\mathrm{PbTiO}_{3}, \mathrm{BaTiO}_{3}, \mathrm{LiNbO}_{3}$, and $\mathrm{SbSl}$ [97]-[101]. Due to equipment limitations of the time, however, it was not possible to measure individual walls directly. Aristov et al. were among the first to spatially resolve domain walls with anomalous electronic properties in ferroelectric $\mathrm{BaTiO}_{3}$ [102] and, later, also in $\mathrm{LiNbO}_{3}$ [103] using scanning electron microscopy (SEM) [104]. The first direct evidence of conducting domain walls was in pioneering experiments by Seidel et al. [94], and since then conductance measurements using conductive atomic force microscopy (cAFM) have been performed on diverse prototypical (proper) ferroelectrics, including $\mathrm{PbZr}_{0.2} \mathrm{Ti}_{0.8} \mathrm{O}_{3}$ [105]-[107], $\mathrm{LiNbO}_{3}$ [108]-[110], and $\mathrm{BaTiO}_{3}$ [111], as well as improper ferroelectrics such as hexagonal $R \mathrm{MnO}_{3}$ [53], [57], [112]-[115], $\mathrm{Cu}_{3} \mathrm{~B}_{7} \mathrm{O}_{13} \mathrm{Cl}$ [116], and (Ca, $\left.\mathrm{Sr}\right)_{3} \mathrm{Ti}_{2} \mathrm{O}_{7}$ [117], demonstrating that domain wall conductivity is a quite general phenomenon. Although conducting domain walls have already been analysed and discussed more than half a century ago, only the recent in-depth studies haverevealed their full technological potential and triggered world-wide interest. During the last decade, domain-wall based multi-configurational devices, atomicscale electronic components and memory technology have been proposed [61], [118], [119]. Another idea is to use domain walls in order to achieve reconfigurable doping: While semiconductor technology enables the precise control of charge dopants during the fabrication process, their location remains fixed. Reconfigurable channels of charge carriers are in principle achievable using polarization charges as quasi-dopants [120]. Then, doping may be achieved in ferroelectrics within the domain walls. For more insight into the physics and properties of ferroelectric domain walls, we refer to, e.g., the recent review articles by Catalan et al. [60], Meier [121], Jiang et al. [119] and Bednyakov et al. [66].

a

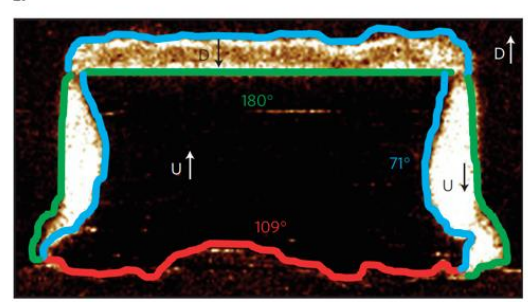

d

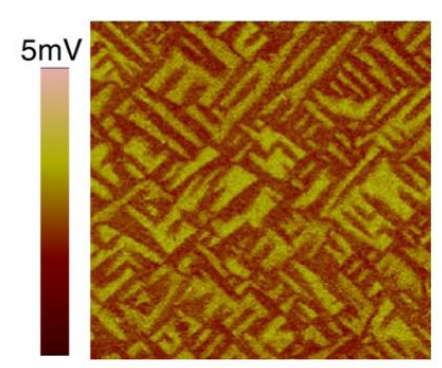

b

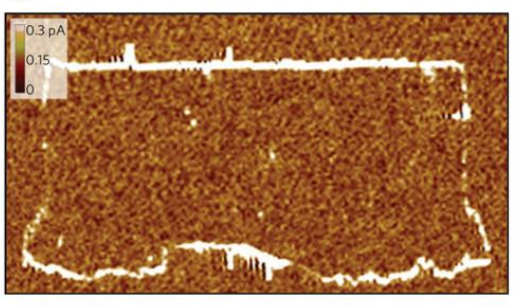

f
C

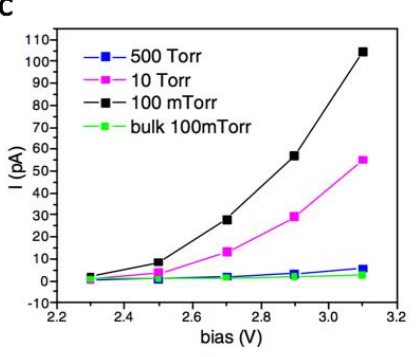

e

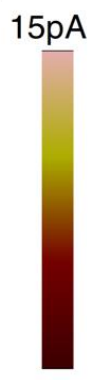

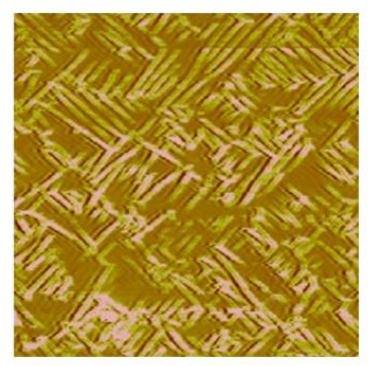

Fig. 5: Conduction at charge-neutral domain walls in multiferroic $\mathrm{BiFeO}_{3}$ thin films. (a) $109^{\circ}, 180^{\circ}$ and $71^{\circ}$ domain walls in La-doped (110) $\mathrm{BiFeO}_{3}$ thin films observed by PFM and the corresponding (b) cAFM map showing enhanced conduction for $180^{\circ}$ and $109^{\circ}$ domain walls (a-b reprinted by permission from Springer Nature, taken from [94]. Copyright 2009 by Springer Nature). (c) Influence of the oxygen pressure after growth on the conduction of $109^{\circ}$ domain walls in $\mathrm{La}$-doped $\mathrm{BiFeO}_{3}$ thin films. (c) is reprinted figure with permission from [90]. Copyright 2010 by the American 
Physical Society. (d) PFM amplitude of (001) $\mathrm{BiFeO}_{3}$ films with a majority of $71^{\circ}$ domain walls and (e) corresponding cAFM image showing enhanced conduction at the domain walls. (f) Arrhenius plot of the current vs. temperature showing a thermally-activated behaviour of the conduction through $71^{\circ}$ domain walls. (d) - (f) from are reprinted figure with permission from [122]. Copyright 2011 by the American Physical Society.

The first direct observation of conductivity at a domain wall was also the first in a multiferroic: Seidel et al. observed room-temperature conduction at $180^{\circ}$ and $109^{\circ}$ domain walls in multiferroic $\mathrm{BiFeO}_{3}$ thin films (Fig. 5a,b), while no conduction could be detected at $71^{\circ}$ domain walls [94]. This seminal paper triggered a plethora of experimental and theoretical works on conductive domain walls partly fuelled by the controversy regarding the origin of these effects. The initial interpretation from Seidel et al., supported by density functional theory (DFT), suggested that electrostatic potential steps at the domain walls were responsible from the enhanced conduction, as well as a reduced bandgap induced by structural transitions in the wall. In addition, DFT calculations showed that these effects were minimized for $71^{\circ}$ domain walls in agreement with experiments. However, following reports [90], [122] concluded that the conduction through these neutral walls was mostly related to extrinsic contributions. Farokhipoor \& Noheda found that $71^{\circ}$ domain walls were also conducting in $\mathrm{BiFeO}_{3}$ thin films (Fig. 5d,e) [122]. Through a temperature analysis of the conduction, they demonstrated that it is governed by thermally activated transport from defects (oxygen vacancies) at low voltage (Fig. 5f) and Schottky emission at higher voltage, just as for domains. The different conduction for domains and domain walls is explained by an increased density of oxygen vacancies at the walls, giving rise to in-gap states and lower electron hopping energy. In the same vein, Seidel et al. reported that the oxygen pressure during the cool-down process after the growth of $\mathrm{La}$-doped $\mathrm{BiFeO}_{3}$ had a large influence on the $109^{\circ}$ domain wall conduction, with a current increase by two orders of magnitude under low oxygen pressure Fig. 5c) [90].

Somewhat easier and more straightforward to understand are electrostatics-driven contributions to the domain wall conductivity. At domain walls where the polarization meets either fully or partially in head-to-head or tail-to-tail configuration (see Fig. 2e,f), positively or negatively domain wall bound charges exist, respectively, creating diverging electrostatic potentials. The compensation of the bound charge can be achieved by the redistribution of mobile charge carriers as we addressed in section 1 . Though charged domain walls are not favoured in proper ferroelectrics, they naturally arise in various multiferroic materials. This abundance is due to the fact that multiferroics are often improper ferroelectrics (see section 1 and 2), where the formation of domains is governed by, e.g., a structural or magnetic order parameter and not the electric polarization [67]. Multiferroic hexagonal manganites, $R \mathrm{MnO}_{3}$, are an interesting example for the natural emergence of charged domain walls: Here, a trimerizing lattice distortion leads to stable $180^{\circ}$ charged domain walls with anomalous electronic transport properties [52],[117]. The extraordinary stability of these charged domain walls is reflected by recent electrostatic force microscopy measurements, which showed that partially unscreened walls arise at low temperature, representing a rare example of a stable, electrically uncompensated oxide interface (Fig. 6c). 
a

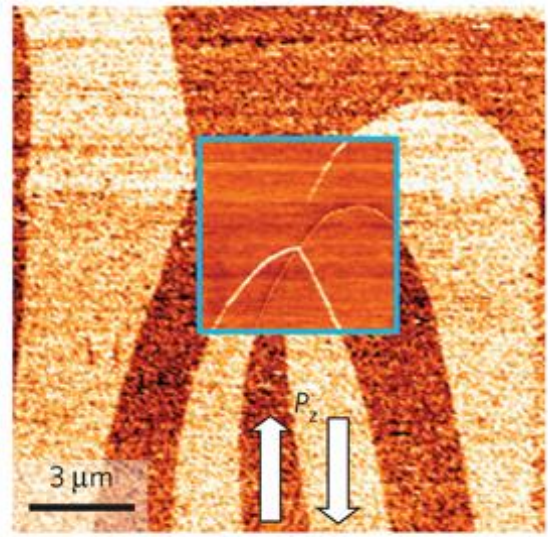

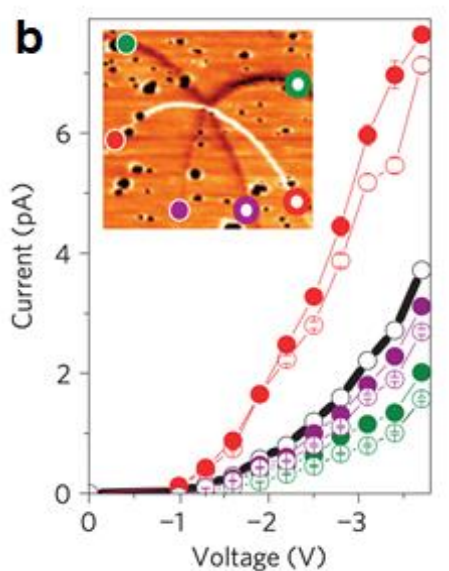

b

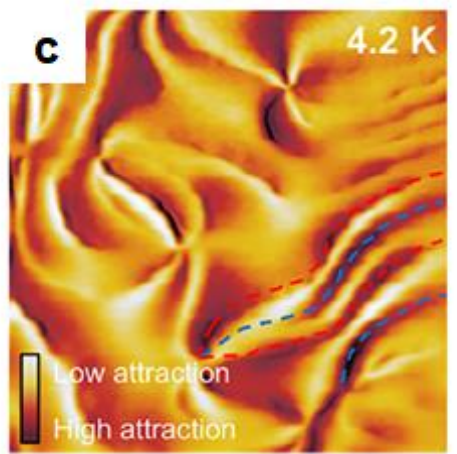

d

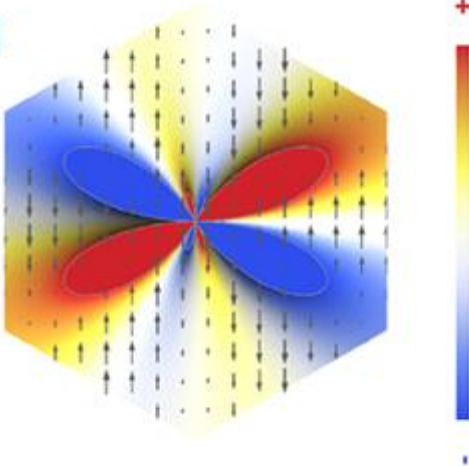

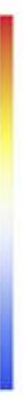

Fig. 6: Charged domain walls in improper ferroelectrics of $\mathrm{ErMnO}_{3}$ hexagonal manganites. (a) PFM image in the yz plane of an $\mathrm{ErMnO}_{3}$ single crystal. The inset shows the cAFM map in the same region. The bright and dark lines show that domain walls have distinct conduction from domains. (b) Local current-voltage characteristics at the locations showed in the inset. Tail-to-tail walls (red) show an enhanced conduction while head-to-head walls (purple and green) are less conducting than the domains (black) (from [53]). (c) Electrostatic force microscopy image of $\mathrm{Er}_{0.99} \mathrm{Ca}_{0.01} \mathrm{MnO}_{3}$ taken at $4.2 \mathrm{~K}$ showing the different fields (imaged as high/low attraction) arising from partially uncompensated charges at domain walls (Reprinted with permission from [124]. Copyright 2019 American Chemical Society). (d) Density plot of the bound-charge distribution emerging from the centre of a vortex core in the hexagonal manganites; the arrows indicate the direction of ferroelectric polarisation. (d) is taken from [22] and reused with permission from ACS. All further permissions must be directed to ACS.

Meier et al. observed that at the characteristic cloverleaf-like ferroelectric domain pattern [57] in $\mathrm{ErMnO}_{3}$ single crystals, the conduction of the domain walls is a continuous function of the wall orientation (Fig. 6a) [53]. Enhanced conductance was found in tail-to-tail walls while head-to-head walls showed a lower conduction than in the domains (Fig. 6b). This can be interpreted by the p-type semiconducting nature of $\mathrm{ErMnO}_{3}$ in which mobile holes are available to screen negative bound charges at tail-to-tail walls. At the same time, the electrostatic potential at the walls shifts the Fermi level into the broad valence band where the effective mass is low, leading to an enhanced conduction of the walls [53]. In order to control and optimize the electronic domain wall properties in hexagonal manganites, effects related to off-stoichiometry [54], [125]-[130] and aliovalent cation substitution on the A- and B-site were studied systematically [131], [132]. Interestingly, recent studies suggest that the emergence of anomalous conductance is not restricted to isolated head-to-head and tail-to-tail walls in $\mathrm{RMnO}_{3}$ : Wherever the walls intersect in the characteristic cloverleaf-like arrangement, ferroelectric vortex cores with emergent U(1) symmetry form [133]-[135]. These vortex cores are quasi-1D objects and exhibit quite unusual bound-charge distributions and electrostatics (Fig. 6d), which - similar to the charged walls - is likely to change the electronic transport properties locally.

The results highlighted here, however, represent only a fraction of the research devoted to the domain and domain wall physics is hexagonal manganites. Due to the stability and abundance of charged domain walls, the material has become an important model system for the theoretical and experimental study of the complex nano-physics of functional domain walls and different application opportunities as nanoscale digital switches and half-wave rectifiers have been proposed [54],[106]. 
The majority of envisioned domain-wall applications in nanoelectronics, such as domain-wall based memories and multi-configurational devices, however, require highly mobile domain walls that can readily be injected and deleted at will. The intentional creation of stable charged domain walls was first demonstrated in 2013 in ferroelectric $\mathrm{BaTiO}_{3}$

a

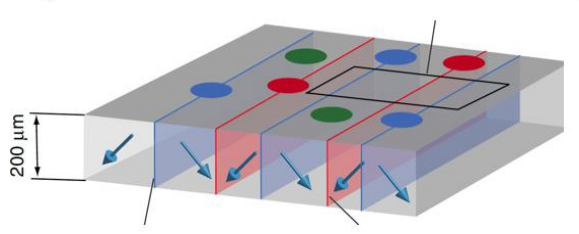

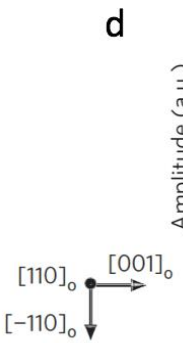

b

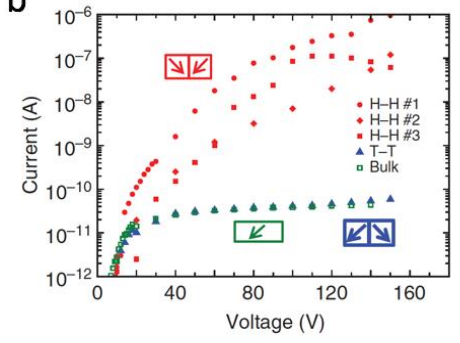

e

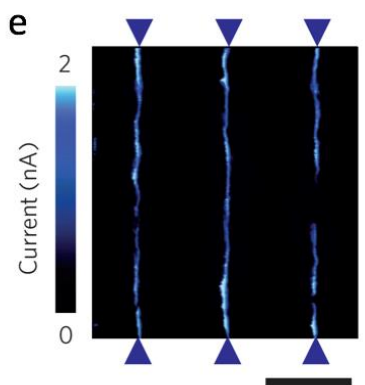

C

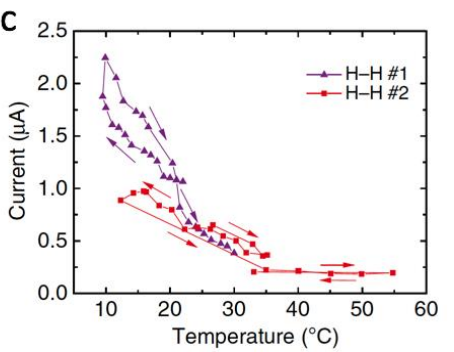

f

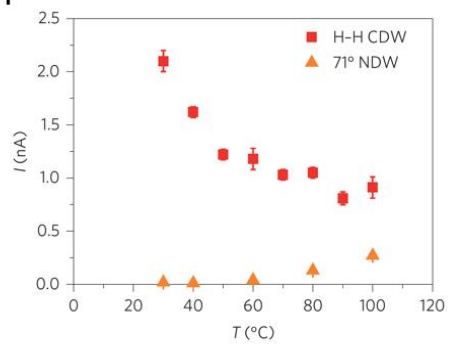

Fig. 7: Conduction at created charged domain walls in ferroelectrics and multiferroics. (a) Sketch of the (110) Ba$\mathrm{TiO}_{3}$ crystal where the surface is covered with $200 \mu \mathrm{m}$ diameter electrodes. The domain walls are irregularly distributed with periods from 100 to $300 \mu \mathrm{m}$. (b) Current-voltage characteristics of the tail-to-tail, head-to-head walls as well as the domains. (c) Temperature dependence of the current through the head-to-head domain walls indicating a metallic behavior. (a) - (c) are reprinted by permission from Springer Nature, taken from [111]. Copyright 2013 by Springer Nature. (d) PFM amplitude of the domain pattern with alternating head-to-head and tail-to-tail domain walls defined by the trailing field of the SPM tip in La-doped $\mathrm{BiFeO}_{3}$ thin films. (e) CAFM maps on the same domain pattern show an enhanced conduction at the head-to-head domain walls. The scale bars in (d) and (e) are $1 \mu \mathrm{m}$. (f) Temperature dependence of the current through the head-to-head walls compared to that of native $71^{\circ}$ domain walls. (d) - (f) are reprinted by permission from Springer Nature, taken from [120]. Copyright 2015 by Springer Nature.

single crystals (Ref. [111]). The stabilization of $90^{\circ}$ charged domain walls was realized by cooling down the single crystal under a strong electric field from above the Curie temperature. Then, the authors compared the conduction of individual head-to-head and tail-to-tail domain walls with the domains by contacting them with 200-micron-wide metallic electrodes (Fig. 7a). While the conduction of tail-to-tail walls was comparable to that of domains, a conduction $10^{4}$ to $10^{6}$ times higher than in the domains was observed in head-to-head domain walls (Fig. 7b) with a metallic character (Fig. $7 \mathrm{c}$ ). However, this frustrated poling process is not technology transferrable for the realization of reconfigurable devices based on ferroelectric and multiferroic thin films. In 2015, Crassous et al. succeeded in creating charged walls in $\mathrm{BaTiO}_{3}$, using the trailing field of the SPM probe tip [136] to design charged ferroelastic domain walls in a multiferroic thin film, this time using La-doped $\mathrm{BiFeO}_{3}$ grown on $\mathrm{DyScO}_{3}(110)$ orthorhombic substrates (Fig. Error! Reference source not found.7d) [120]. CAFM maps of the artificial domains revealed a remarkable enhancement of the conductivity at headto-head domain walls only (Fig. 7e) with an unprecedentedly high currents (two orders of magnitude higher than previous reports with neutral walls). Moreover, temperature-dependent measurements indicated a metallic behaviour for these charged walls as opposed to the thermally-activated behaviour of neutral $71^{\circ}$ domain walls (Fig. 7f). On the other hand, tail-to-tail ferroelastic walls do not show a significant conduction as this configuration cannot be compensated by mobile holes, which gives rise to a roughening of the wall as well. In addition, the authors demonstrated the reconfigurability of the writing process as well as the nanoscale manipulation of conductive head-to-head walls.

\subsection{Magnetism at domain walls}

In $\mathrm{BiFeO}_{3}$, the coupling between ferroelectric and antiferromagnetic domain walls has been extensively investigated [137]-[139]. In $\mathrm{BiFeO}_{3}$ thin films grown under high compressive strain, the rhombohedral-like phase and the tetragonallike phase coexist in the form of nanoscale structures. Using XMCD-PEEM, Zhang et al. observed an enhancement of 
magnetism at the nanoscale boundaries of these mix-phase thin films [140]. The magnetotransport properties of these walls have also been investigated [141].

Domain wall magnetism and its correlation to the ferroelectric order was first investigated in hexagonal manganites. These materials order antiferromagnetically below a Néel temperature, $\mathrm{T}_{\mathrm{N}} \approx 100 \mathrm{~K}\left(\mathrm{~T}_{\mathrm{C}} \approx 1000 \mathrm{~K}\right.$, type I multiferroic
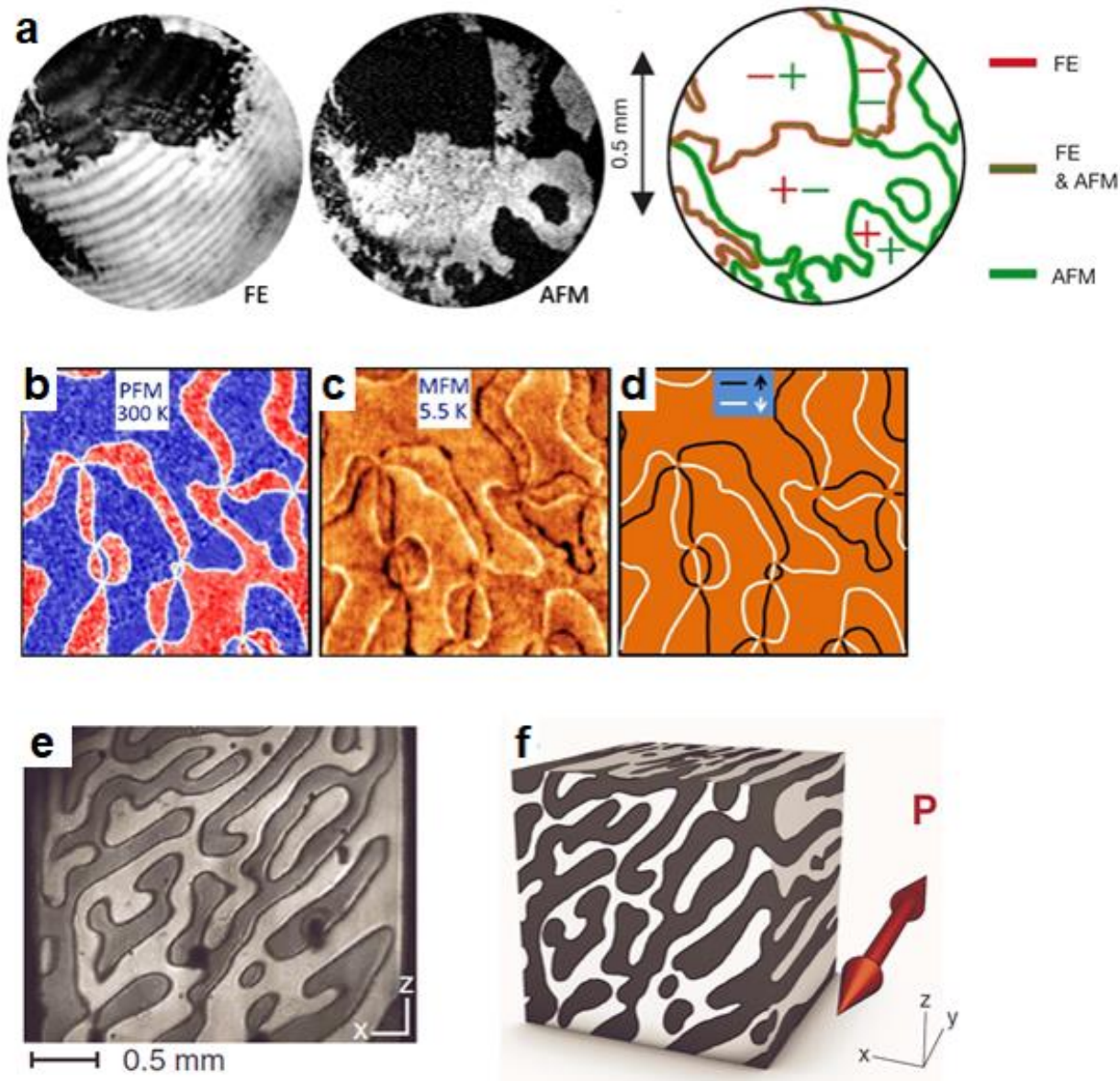

Fig. 8: Electric and magnetic domains in multiferroics. (a) Coexistence of ferroelectric and antiferromagnetic domains in hexagonal $\mathrm{YMnO}_{3}$ visualized by SHG. Bright and dark areas correspond to regions with different orientations of the order parameter ( $P$ on the left image - ferroelectric (FE), $L$ on the right image - antiferromagnetic (AFM)). The sketch on the right shows that some of the domain walls are both antiferromagnetic and ferroelectric. (a) is reprinted by permission from Springer Nature, taken from [86]. Copyright 2002 by Springer Nature. (b) PFM image of hexagonal $\mathrm{ErMnO}_{3}$ showing up and down ferroelectric domains. (c) MFM image at low temperature at the same location as in (b) showing an induced magnetic moment at ferroelectric domain walls. (d) Sketch from image c emphasizing the magnetic moments at domain walls of the $16 \times 16 \mu \mathrm{m}^{2}$ image. (b)-(d) reprinted (adapted) with permission from [142]. Copyright 2012 American Chemical Society [142]. (e) Multiferroic domain walls in $\mathrm{MnWO}_{4}$ visualized by SHG. (f) Illustration showing the 3D distribution of the multiferroic domains in (e). (e) - (f) Reprinted with permission from [37]. Copyright 2009 by the American Physical Society.

[62],[137]). Using SHG imaging at $6 \mathrm{~K}$, Fiebig et al. were able to discriminate antiferromagnetic domains from ferroelectric domains in a $\mathrm{YMnO}_{3}$ single crystal [86]. They found that ferroelectric domain walls always coincide with antiferromagnetic domain walls, while antiferromagnetic domain walls can also exist independently (Fig. 8a). Although the resolution of the applied SHG experiment $(\gtrsim 1 \mu \mathrm{m})$ did not allow to resolve the domain walls directly, this was the first experimental evidence of multiferroic domain walls. While no magnetoelectric coupling exists in the bulk, the coupling of the two ferroic orders suggests a possible magnetoelectric coupling at domain walls. In 2012, Geng et al. studied the magnetic properties of multiferroic domain walls in an $\mathrm{ErMnO}_{3}$ single crystal using low-temperature MFM [142]. They first used PFM to image the ferroelectric domain structure at room temperature (Fig. 8b) and the MFM data showed that each multiferroic domain wall carried a net magnetization (Fig. 8b-c), which they attributed to the presence of uncompensated $\mathrm{Er}^{3+}$ spins at the domain walls [142], [143]. 
Multiferroic domains and domain walls with explicitly strong coupling between the electric and magnetic order were discovered in the spin-spiral multiferroic $\mathrm{MnWO}_{4}$ (type II) [37], [93]. In $\mathrm{MnWO}_{4}$, hybrid ferroelectric/antiferromagnetic domains arise below $15 \mathrm{~K}$ (Fig. 8e,f) driven by an elliptical spin spiral that breaks the inversion symmetry and, thereby, induces improper ferroelectricity. Because of the magnetic origin of the ferroelectric order, and in strong contrast to the hexagonal manganites, electric and magnetic domain walls always coexist in $\mathrm{MnWO}_{4}$. The inner structure of these walls remains to be measured, but theory predicts that they are antiferromagnetic in nature, similar to the case illustrated in Fig. 4c [36].

Recently, Farokhipoor et al. reported enhanced magnetism at domain walls in epitaxial thin films of the orthorhombic spin-spiral multiferroic $\mathrm{TbMnO}_{3}$ grown on $\mathrm{SrTiO}_{3}$ (Ref. [144]). They found out that the macroscopic magnetic signal of the samples is correlated to the density of domain walls of $\mathrm{TbMnO}_{3}$ with different thicknesses. While the results are supported by DFT calculations, direct evidence of magnetism at domain walls remains to be demonstrated with microscopy techniques. Another idea is the emergence of dynamical magnetic fields at moving ferroelectric walls proposed by Juraschek et al., which extends magnetism in domain walls into the realm of dynamical multiferroicity, foreshadowing exciting perspectives for future experimental work on multiferroic domain walls [145].

\section{Manipulating domains and domain walls}

\subsection{Electric control of antiferromagnetic domains with $\mathrm{BiFeO}_{3}$}

$\mathrm{BiFeO}_{3}$ has been involved in a number of scientific breakthroughs, such as the observation conducting ferroelectric domain walls [94] and room temperature electric field control of magnetism [146]. It is thus not surprising that research efforts on multiferroic domains and domain walls have been highly concentrated on this material during the last fifteen years, especially in thin films (DOI:10.1515/PSR.2019.0070). A primary goal of magnetoelectric multiferroics was to control magnetic domains via the low power electric-field switching of ferroelectric domains in view of possible applications in spintronics. $\mathrm{BiFeO}_{3}$ being an antiferromagnetic ferroelectric, concepts based on the electric control of exchange bias of an adjacent ferromagnet were proposed [147].

In 2014, Heron et al. investigated the kinetics of the polarization switching process in 100 -nm-thick $\mathrm{BiFeO}_{3}$ thin films grown on $\mathrm{DyScO}_{3}(110)$ substrates [148]. Using time-dependent PFM, they found out that the out-of-plane electric field was giving rise to a $180^{\circ}$ reversal of polarization in a two-step switching process combining $71^{\circ}$ and $109^{\circ}$ switching (Fig. 9a). Ab initio calculations supported these observations with a direct $180^{\circ}$ switching having a too high energy barrier compared to the two-step process. In addition, it was predicted that the two-step switching enables an in-plane switching of the canted moment by $180^{\circ}$ through the reversal of the rotation of the oxygen octahedra (Fig. $9 \mathrm{~b}$ ). This was experimentally confirmed by visualizing the influence of the ferroelectric switching onto the magnetization of a $\mathrm{Co}_{0.9} \mathrm{Fe}_{0.1}$ ferromagnet deposited on the film of $\mathrm{BiFeO}_{3}$. Using XMCD-PEEM, the authors observed that, after the application of a $6 \mathrm{~V}$ voltage pulse to switch the ferroelectric polarization, the net magnetization of the ferromagnet was reversed (Fig. 9c).

However, these experiments consider that $\mathrm{BiFeO}_{3}$ is in a canted G-type antiferromagnetic phase [149] in contradiction with the measurements of a spin cycloid in low-strained $\mathrm{BiFeO}_{3}$ films grown on substrates such as $\mathrm{DyScO}_{3}(110)$ using Mossbauer and Raman spectroscopies [83]. Using NV magnetometry, Gross et al. recently confirmed that this state was favoured by imaging in real-space the stray field coming from the spin-cycloid of $\mathrm{BiFeO}_{3}$, with an imprint of the ferroelectric stripe-domains onto the magnetic texture (Fig. 3h) [78]. In addition, by defining single ferroelectric domains using the trailing field of the SPM tip, they observed a single spin cycloid within the plane of the film (Fig. 10a). The spin cycloid period is about $70 \mathrm{~nm}$ and quantitative NV magnetometry indicates that both the magnetoelectric and the Dzyaloshinskii-Moriya interactions play a role, resulting in a wiggling cycloid (Fig. 10b). Hence, thanks to the magnetoelectric exchange coupling in $\mathrm{BiFeO}_{3}$, the propagation vector of the spin cycloid can be controlled deterministically, envisioning potential applications in the field of magnonics [150] or antiferromagnetic spintronics [151]. Besides, the exchange coupling between a strong ferromagnet and a non-collinear antiferromagnetic multiferroic should be investigated more thoroughly both theoretically and experimentally.

For large epitaxial strain on $\mathrm{BiFeO}_{3}$, the spin cycloid order is destabilized and a canted G-type antiferromagnetic order results in $\mathrm{BiFeO}_{3}$ (Ref. [83]). Using SHG, Chauleau et al. observed that two types of submicron antiferromagnetic domains coexist within a single $10 \times 10 \mu \mathrm{m}^{2}$ ferroelectric domain of $\mathrm{BiFeO}_{3}$ grown on $\mathrm{SrTiO}_{3}(001)$ (Fig. 3Error! Reference source not found.j). In addition, they were able to manipulate these antiferromagnetic domains with multiple 
stimuli (Fig. 10c). First, manipulating the ferroelectric domains gave rise to a new pattern of antiferromagnetic domains. This is because, due to magnetoelectric coupling, switching the polarization variants toggles the direction of magnetic anisotropy, which can induce a rotation of the antiferromagnetic vector. Then, they heated the $\mathrm{BiFeO}_{3}$ sample close to the Néel temperature, which led to a reinitialization of the antiferromagnetic domains. They showed that sub-coercive electric fields were also manipulating these antiferromagnetic domains without changing the ferroelectric domain pattern. Finally, $100-\mathrm{fs}$ laser pulses were used to generate a terahertz electrical pulse in the sample. A profound modification of the antiferromagnetic domain pattern resulted, as this matches the range of the antiferromagnetic resonance in $\mathrm{BiFeO}_{3}$. Thus, one can envisage controlling the antiferromagnetic order in a contactless manner using ultrafast light pulses for an all-optical information technology approach.

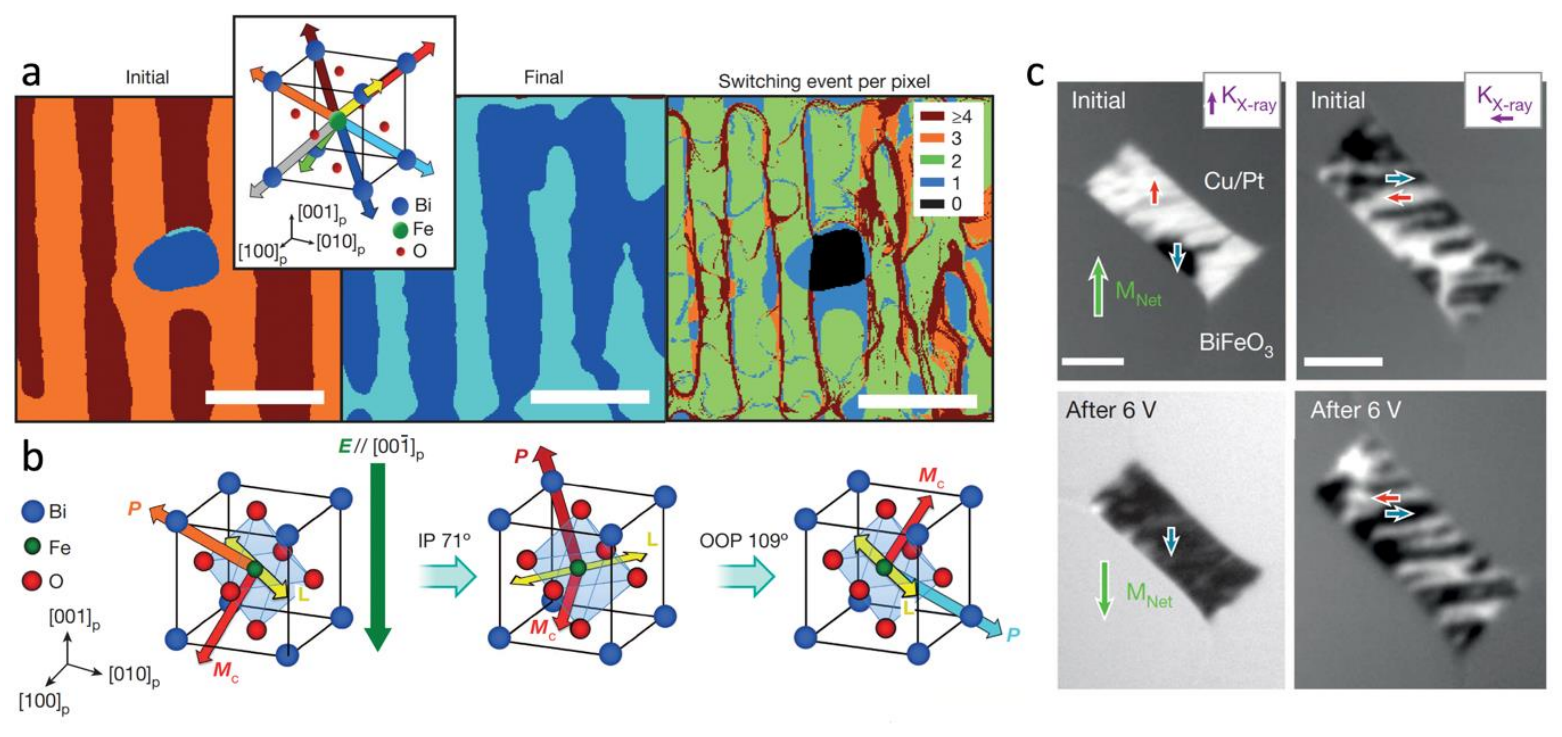

Fig. 9: Deterministic switching of magnetization with an electric field. (a) Polarization vector images determined from PFM measurements before (initial) and after applying an out-of-plane field (final) to the 100 -nm-thick $\mathrm{BiFeO}_{3}$ grown on $\mathrm{SrRuO}_{3} / \mathrm{DyScO}_{3}(110)$. On the right, image of the number of switching events per pixel, showing that in average a two-step switching is favored. (b) Schematic of the two-step $180^{\circ}$ switching of polarization (P) with a $71^{\circ}$ and a subsequent $109^{\circ}$ switching. The antiferromagnetic vector $(\mathrm{L})$ and canted moment $\left(\mathrm{M}_{\mathrm{C}}\right)$ are also represented. Consequently, the two-step switching is accompanied by a $180^{\circ}$ rotation of the canted moment of $\mathrm{BiFeO}_{3}$. (c) $\mathrm{XMCD}$-PEEM images of the in-plane moment of a $\mathrm{Co}_{0.9} \mathrm{Fe}_{0.1}$ layer deposited on $\mathrm{BiFeO}_{3}$ with components viewed perpendicular (vertical $\mathrm{K}_{\mathrm{X} \text {-ray }}$, where $\mathrm{K}_{\mathrm{X} \text {-ray }}$ defines the in-plane component of the incident $\mathrm{X}$-ray beam) and parallel to the stripe domains (horizontal $\mathrm{K}_{\mathrm{X} \text {-ray }}$ ). The directions of the magnetizations in each domain are highlighted with blue and red arrows, which correspond to the local moment direction being perpendicular or parallel to $\mathrm{K}_{\mathrm{X} \text {-ray. }}$. The net $\mathrm{Co}_{0.9} \mathrm{Fe}_{0.1}$ magnetization (green arrows) reverses after the voltage is applied. Figure is reprinted by permission from Springer Nature, all panels taken from [148]. Copyright 2014 by Springer Nature.

Although a significant fraction of current research on the manipulation of multiferroic domains and domain walls focuses on $\mathrm{BiFeO}_{3}$-based systems, it is important to note that there are other systems which have displayed direct switching of the ferroelectric state under applied magnetic fields at room temperature, such as lamellae of lead zirconium titanate (PZT)-lead iron tantalate (PFT) [152]-[155]. In this system, there are direct images, via PFM, of magnetic-field induced changes to the ferroelectric domain structure [156], the equivalence of (sign dependent) magnetic and electric fields to switch the ferroelectric domain structure [157], the appearance of the phase transitions in all three order parameters when they are measured independently [158], and measurements of changes in capacitance as a function of magnetic field [159]. The reason why large coupling constants have been observed in this family of materials is likely to be due to the low structural energy costs of switching between ferroelectric states, evidenced by the relaxor like domain pattern sometimes observed in thin TEM samples [156] and the small variation in lattice parameters [160]. 
a
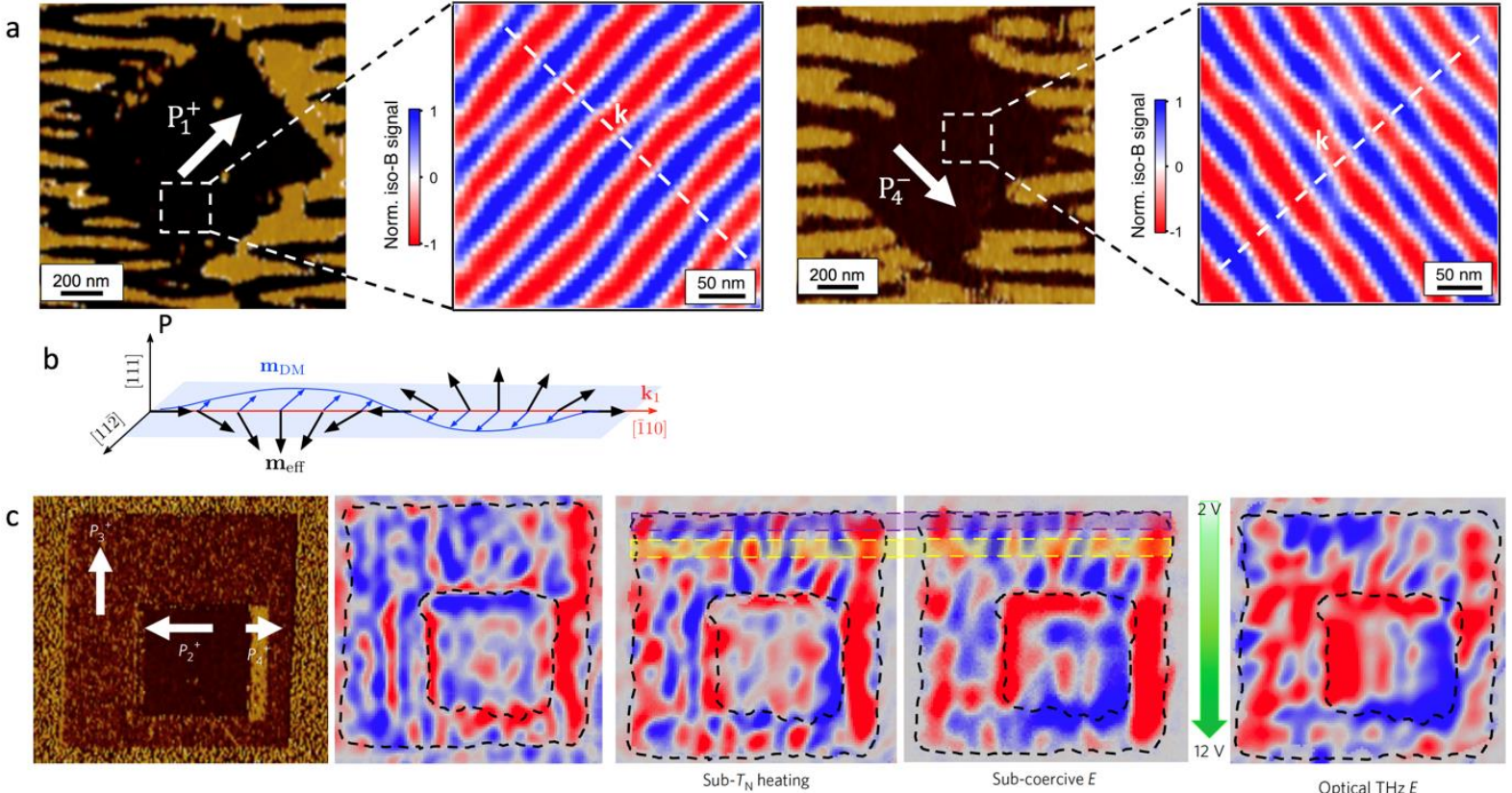

Fig. 10: Manipulation of the antiferromagnetic order in $\mathrm{BiFeO}_{3}$ thin films. (a) Micron square single domain in $\mathrm{BiFeO}_{3}$ grown on $\mathrm{DyScO}_{3}(110)$ read by in-plane PFM and corresponding $\mathrm{NV}$ magnetometry image in the center of this square showing the periodic magnetic signal of a spin cycloid. On the right, another micron square single domain with the corresponding magnetic image showing that the propagation vector of the spin cycloid can be rotated by $90^{\circ}$ via the magnetoelectric coupling. (b) Sketch of the wiggling cycloid in $\mathrm{BiFeO}_{3}$ with two corresponding canted moments $\mathrm{m}_{\text {eff }}$ and $\mathrm{m}_{\mathrm{DM}}$ corresponding to magnetoelectric and Dzyaloshinskii-Moriya interactions, respectively. (a) - (b) are reprinted by permission from Springer Nature, taken from [78]. Copyright 2017 by Springer Nature. (c) In-plane PFM of a $\mathrm{BiFeO}_{3}$ film grown on $\mathrm{SrTiO}_{3}(001)$ in which three domains were defined in a $10 \times 10 \mu \mathrm{m}^{2}$ square. On the right corresponding SHG images and manipulation with temperature $(570 \mathrm{~K})$, electric field $(2$ to $10 \mathrm{~V})$ and optical THz. (c) is reprinted by permission from Springer Nature, taken from [79]. Copyright 2017 by Springer Nature.

\subsection{Magnetic control of domain wall charge states in improper ferroelectrics}

Spin-driven multiferroics develop domains and domain walls with inseparably entangled electric and magnetic degrees of freedom as introduced in sections 1 and 2 (see DOI:10.1515/PSR.2019.0016 for an extended discussion on spindriven multiferroics). The strong magnetoelectric coupling represents a unique feature of this material class, offering interesting opportunities for the manipulation of domain walls and their electronic properties, such as proof-of-concept work on iron garnet, where the electrical polarity of domain walls was switched with a magnetic field [161]; or in more recent work, where a magnetic field was applied along the $\mathrm{b}$ axis of the spin-driven multiferroic $\mathrm{Mn}_{0.95} \mathrm{Co}_{0.05} \mathrm{WO}_{4}$, to continuously rotates the magnetic easy plane by $90^{\circ}$ and with it the orientation of the ferroelectric polarization (Fig. 11a) [36]. Leo et al. exploited this phenomenon to gain control of the domain wall charge state. They used SHG to image the ferroelectric spin-spiral domains in a $\mathrm{Mn}_{0.95} \mathrm{Co}_{0.05} \mathrm{WO}_{4}$ single crystal and created neutral $180^{\circ}$ ferroelectric domain walls by applying an electric field through macroscopic electrodes (Fig. 11b, top). As the primary magnetic order prevents the motion of the wall, a magnetic field of $6 \mathrm{~T}$ was used to switch the polarization by $90^{\circ}$ without changing the location of the domain wall (Fig. 11b, bottom). This way, a magnetic-field-driven change of the charge state was achieved switching from electrically neutral to positively/negatively charged walls [36]. Landau-Lifshitz-Gilbert simulations support the observations of the magnetic-field-induced change of the ferroelectric domain wall charge and give additional insight into the complex Néel-type structure of the domain walls (Fig. 4c).

A similar but discontinuous effect was observed for domain walls in $\mathrm{TbMnO}_{3}$ [39]. In orthorhombic $\mathrm{TbMnO}_{3}$, a spin-cycloid appears below $\mathrm{T}_{\mathrm{C}}=27 \mathrm{~K}$ which breaks inversion symmetry and leads to a spontaneous polarization along the $\mathrm{c}$ axis [48]. Applying a magnetic field along the $\mathrm{b}$ axis alters the orientation of the magnetic easy plane, giving rise to a polarization flop to the a axis through a first-order phase transition (Fig. 11c) [48], [162]. Using SHG, Matsubara et al [39]. observed the evolution of ferroelectric domains in a $\mathrm{TbMnO}_{3}$ single crystal under the application of an electric field along the $\mathrm{c}$ axis. The crystal is naturally forming neutral $180^{\circ}$ domain walls elongated along the $\mathrm{c}$ axis (Fig. 9d, top). As the magnetic exchange interaction is also stronger along c, both magnetic and electric energies are lowered by the formation of these walls. Then they used SHG at $9 \mathrm{~K}$ to track the evolution of domains with polarization parallel to 
$\mathrm{a}$ and $\mathrm{c}$ under a magnetic field of $10 \mathrm{~T}$ applied along the $\mathrm{b}$ axis. Within the resolution of the technique (a few microns), they did not detect any change in the domain pattern from 0 to $10 \mathrm{~T}$ while the polarization flopped from $\mathrm{c}$ to a (Fig. 11d, bottom). This implies that the walls changed from a neutral side-by-side configuration to either tail-to-tail or head-tohead charged states. LLG simulations concluded that the magnetic field along b exerts an effective torque on the magnetization around this axis and leads to an identical flop for both up or down polarization previously aligned along c. The deterministic nature of this multiferroic phase transition allows to convert neutral walls into charged domain walls.

The opportunity to magnetically convert neutral domain walls into charged domain walls is intriguing as it allows, in principle, reversible control of the density of screening charges and, hence, conductivity. This would enable the design of nano-sized electrical gates, where ON and OFF states are set by the magnetic field. Going beyond the application of external magnetic (or electric) fields for controlling the domain wall charge state, intense light fields have been used to selectively create neutral and charged walls in spin-driven multiferroics. Manz et al. showed that both neutral and charged walls can be written and erased in $\mathrm{TbMnO}_{3}$ using pulsed or continuous wave-lasers (Fig. 11e-f) [163]. The findings demonstrate the possibility to optically engineer individual domain walls or patterns of higher complexity as required, e.g., for domain-wall based circuitry. At present, however, any technological merit is clearly suspended by the cryogenic range of the multiferroic phase in spin-spiral multiferroics such as $\mathrm{TbMnO}_{3}$ and $\mathrm{Mn}_{0.95} \mathrm{Co}_{0.05} \mathrm{WO}_{4}$ and a oneto-one correlation between domain wall charge state and local conductivity is yet to be demonstrated in these materials. Still, the conceptual results reveal completely new pathways for domain wall engineering and highlight the additional functionalities that arise from the strong coupling between electric and magnetic order available in multiferroics.

a

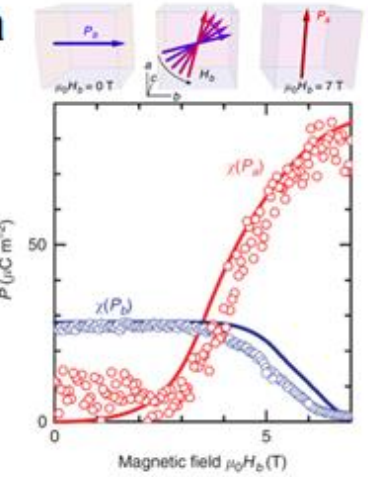

e

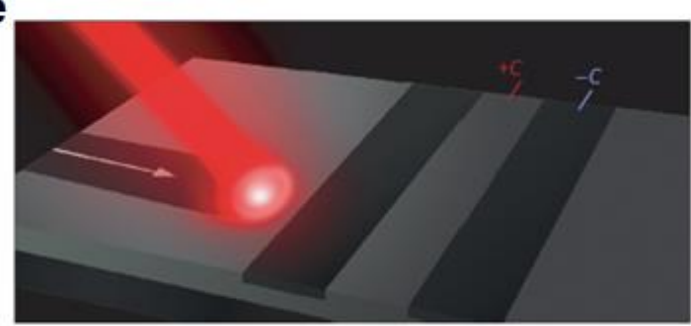

b

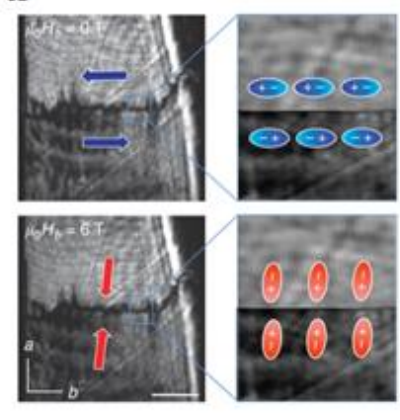

c

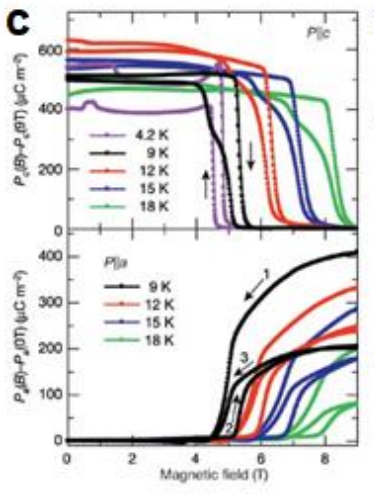

f

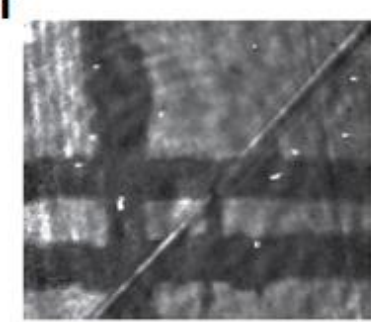

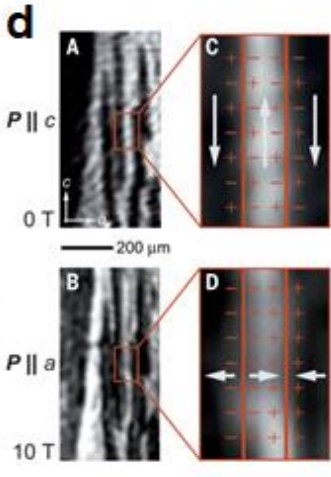

g

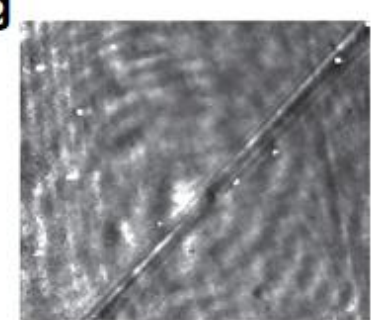

Fig. 11: Magnetic control of the domain wall charge in type II multiferroics. (a) Top, sketch illustrating the magneticfield-induced polarization re-orientation from the $\mathrm{b}$ to the a axis in $\mathrm{Mn}_{0.95} \mathrm{Co}_{0.05} \mathrm{WO}_{4}$. Bottom, pyroelectric current and integrated SHG measurements at $5 \mathrm{~K}$ showing the magnetic field induced rotation of polarization. (b) Top, spatiallyresolved SHG of a side-by-side $180^{\circ}$ domain wall prepared by applying an electric field along b at zero magnetic field. Bottom, SHG image on the same area under a magnetic field of $6 \mathrm{~T}$ showing that the wall did not move and becomes a tail-to-tail wall with polarization along a. The scale bars is $250 \mathrm{~mm}$. (a) - (b) are reprinted by permission from Springer Nature, taken from [36]. Copyright 2015 by Springer Nature. (c) Electric polarization flop in $\mathrm{TbMnO}_{3}$ with a magnetic field applied along a and c axis. (c) is reprinted by permission from Springer Nature, taken from [48]. Copyright 2003 by Springer Nature. (d) Domain structure of $\mathrm{TbMnO}_{3}$ across the first-order polarization flop observed with SHG. Top, ferroelectric domain structure in the ground state. Bottom, the application of a magnetic field of $10 \mathrm{~T}$ flops the polarization along the a axis but the domain structure does not change, implying that the walls become charged. (d) is taken from [39]. Reprinted with permission from AAAS. (e) Schematic image of the optical poling procedure used by Manz et al. [163] to reversibly write antiferromagnetic domains on $\mathrm{TbMnO}_{3}$. (f) SHG image showing laser-written antiferromagnetic domains with positive $(+\mathrm{C}$, bright $)$ and negative $(-\mathrm{C}$, dark) spin chirality. $(\mathrm{g})$ SHG image of the same region shown in $\mathrm{f}$ after erasing the laser-written $-\mathrm{C}$ domains again. $(\mathrm{e})-(\mathrm{g})$ are reprinted by permission from Springer Nature, taken from [163]. Copyright 2016 by Springer Nature. 


\subsection{Tailoring topological states in multiferroics}

The ability to electrically write ferroelectric domain patterns with a conductive tip [136] offers exciting possibilities to realize and harness configurations beyond domain walls that will show topological properties in the definition given in section 2 (i.e. possessing an integer or semi-integer topological charge). The most classical topological textures by this definition are vortices $(Q=1 / 2)$ and skyrmions $(Q=1)$ (antivortices have $Q=-1 / 2$ and antiskyrmions $Q=-1)($ see also DOI:10.1515/PSR.2019.0017). So far, research has concentrated mostly on topological electric structures.

After early theoretical predictions by Naumov et al. [164], ferroelectric vortices were first observed in microscale capacitors of PZT by Gruverman et al. in 2008 (Ref. [165]). Since then there have been numerous reports in various types of systems (mostly $\mathrm{Pb}(\mathrm{Zr}, \mathrm{Ti}) \mathrm{O}_{3}, \mathrm{BaTiO}_{3}$ and $\mathrm{BiFeO}_{3}$ ). Two main strategies have been pursued, namely: (i) nanostructuration by, e.g., self-organized growth (12a-c) [166], [167], deposition through sacrificial templates [168]; and (ii) electric-field manipulation by scanning probe tips [169]. Only in a few of these studies has the
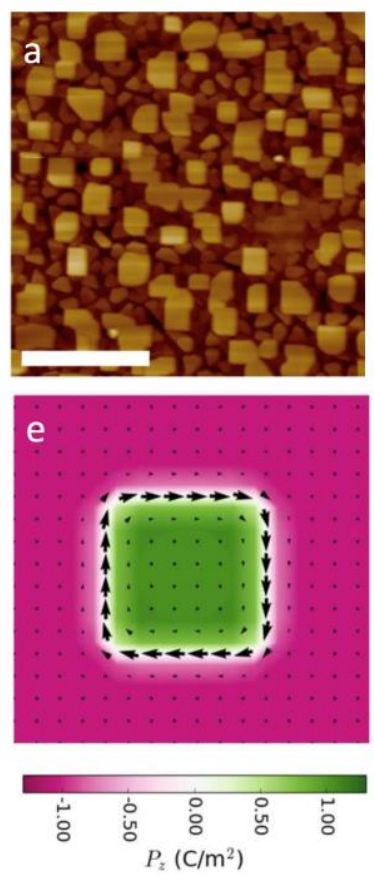

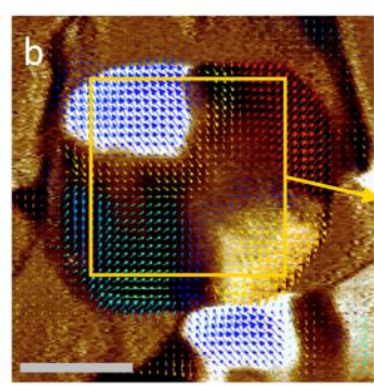

f

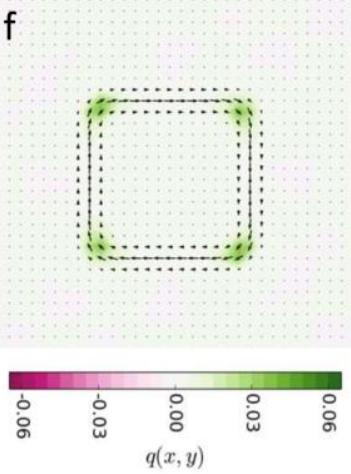

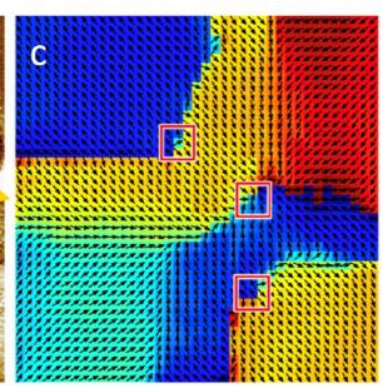

g

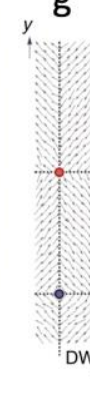

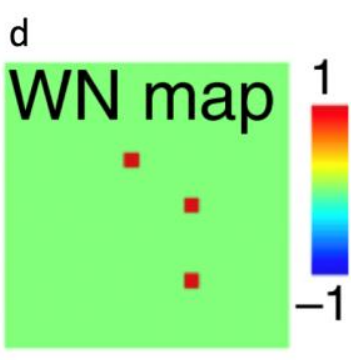

h

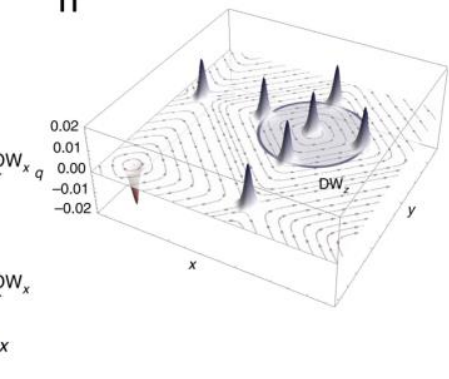

Fig. 12: Topological domain structure in multiferroics. (a) Atomic force microscopy image of a $\mathrm{BiFeO}_{3}$ film on $\mathrm{LaAlO}_{3}$ with self-assembled nanoplates. (b) Vector maps of in-plane piezoresponse overlaid on the corresponding outof-plane PFM contrast of one such nanoplate. (c) In-plane piezoresponse angle maps extracted from the yellow box in (b). (d) Winding number (WN) maps determined from (c). (a)-(d) are reprinted by permission from Springer Nature, taken from [167]. Copyright 2018 by Springer Nature. Calculated (e) polarization and (f) Pontryagin density of a nanodomain in $\mathrm{PbTiO}_{3}$ (e-f reprinted with permission of AAAS from [170] (C) The Authors, some rights reserved; exclusive licensee American Association for the Advancement of Science. Distributed under a Creative Commons Attribution NonCommercial License 4.0 (CC BY-NC) http://creativecommons.org/licenses/by-nc/4.0/). (g) Vortex structure of a $\mathrm{BaTiO}_{3}$ nanowire embedded in $\mathrm{SrTiO}_{3}$. (h) Associated Pontryagin density map. (g)-(h) are reprinted by permission from Springer Nature, taken from [171]. Copyright 2015 by Springer Nature.

topological character of these vortices been explicitly quantified, for instance by computing the vorticity from vectorPFM maps (Fig. 12d) or phase-field simulations mimicking the observed polarization textures. Some works report the electrical manipulation of these vortices, paving the way towards their use in nanoelectronics. Ferroelectric skyrmions were predicted to be stable in nanocomposites (Fig. 12g-h) [171] and nanodomains (Fig. 12e-f) [170] and, most recently, their existence has been confirmed experimentally in ferroelectric superlattices of $\left(\mathrm{PbTiO}_{3}\right) \mathrm{n} /(\mathrm{SrTiO}) \mathrm{n}$ [172]. To date, whether these ferroelectric topological structures translate into magnetic ones through the local magnetoelectric coupling remains elusive but new high-sensitivity magnetic microscopy techniques such as NV microscopy may shed light on this issue.

In metallic systems with magnetic skyrmion structures, the topological charges can influence electron transport to produce a topological Hall effect. Many questions arise regarding the consequence of the presence of electric and/or 
magnetic topological textures in multiferroics in view of their very low conductivity, practically hampering Hall measurements, and more generally on the influence of an electrical topological charge on the macroscopic physical properties. Several papers have predicted other types of phenomena arising from the presence of magnetic topological charges, such as the appearance of a topological orbital moment [173], [174] or a spin-chirality-driven inverse Faraday effect [175]. The mounting interest for topological structures in ferroelectric now calls for similar predictions of exotic effects, driven by electrical topology.

\section{Conclusions}

In this article, we reviewed the recent developments in the field of domains and domain walls in multiferroics. More complete review articles dedicated to domain wall nanoelectronics [60], [66], [176] or functional domain and domain walls in multiferroics [67], [68], [121] can be found elsewhere. Charged domain walls have emerged within the last few years as nanoscale objects with enhanced conductivity or distinct magnetism. They are found naturally in improper ferroelectrics [53] or designed in multiferroics by scanning probe microscopy [120], which opens the way towards reconfigurable nanocircuitry [30], [60] and on-demand spintronic nanocomponents.

New tools are now available to image magnetic textures in antiferromagnets at the local scale. These techniques could help understanding the interplay between ferroelectricity and magnetism in multiferroic domain walls. In addition, the field of antiferromagnetic spintronics [151] could be extended to multifunctional materials such as multiferroics, in which the propagation of spin waves [177] could be controlled by the electric control of ferroelectric domains.

Finally, the possibility to design topological states [178] with electric-field input is particularly exciting for multiferroics. Theoretical works suggest the possibility to fabricate electric analogy of magnetic skyrmions. In addition, recent observations reveal the presence of non-Ising walls in $180^{\circ}$ domain walls of regular ferroelectrics $\left(\mathrm{Pb}(\mathrm{Zr}, \mathrm{Ti}) \mathrm{O}_{3}\right.$ and $\mathrm{LiTaO}_{3}$ ) [35]. This indicates that complex interconnected chiral electric and magnetic structures could be realized in multiferroics.

\section{References}

[1] H. Schmid, "Multi-ferroic magnetoelectrics," Ferroelectrics, vol. 162, no. 1, pp. 317-338, 1994.

[2] A. K. Tagantsev, L. E. Cross, and J. Fousek, Domains in ferroic crystals and thin films. Springer-Verlag New York, 2010.

[3] A. Hubert and R. Schäfer, Magnetic Domains The Analysis of Magnetic Microstructures. Springer-Verlag Berlin Heidelberg, 1998.

[4] J. C. Tolédano and P. Tolédano, The Landau Theory of Phase Transitions, vol. 3. World Scientific, 1987.

[5] V. K. Wadhawan, "Ferroelasticity," Bull. Mater. Sci., vol. 6, no. 4, pp. 733-753, 1984.

[6] D. Fong et al., "Stabilization of Monodomain Polarization in Ultrathin $\mathrm{PbTiO}_{3}$ Films," Phys. Rev. Lett., vol. 96, no. 12, p. 127601, Mar. 2006.

[7] A. Schilling, S. Prosandeev, R. G. P. McQuaid, L. Bellaiche, J. F. Scott, and J. M. Gregg, "Shape-induced phase transition of domain patterns in ferroelectric platelets," Phys. Rev. B - Condens. Matter Mater. Phys., vol. 84, no. 6, p. 64110, Aug. 2011.

[8] A. Schilling et al., "Domains in ferroelectric nanodots," Nano Lett., vol. 9, no. 9, pp. 3359-3364, Sep. 2009.

[9] S. Kalinin and A. Gruverman, Scanning probe microscopy, vol. 2. New York: Springer US, 2007.

[10] E. A. Eliseev, Y. M. Fomichv, S. V. Kalinin, Y. M. Vysochanskii, P. Maksymovich, and A. N. Morozovska, "Labyrinthine domains in ferroelectric nanoparticles: Manifestation of a gradient-induced morphological transition," Phys. Rev. B, vol. 98, no. 5, p. 54101, Aug. 2018.

[11] F. Gilletta, "Evolution of ferroelectric domains in TGS single crystals," Phys. Status Solidi, vol. 11, no. 2, pp. 721-727, Jun. 1972.

[12] W. J. Merz, "Double hysteresis loop of BaTiO3 at the curie point," Phys. Rev., vol. 91, no. 3, pp. 513517, Aug. 1953.

[13] C. Kittel, "Physical theory of ferromagnetic domains," Rev. Mod. Phys., vol. 21, no. 4, pp. 541-583, Oct. 1949.

[14] C. Kittel, "Theory of the structure of ferromagnetic domains in films and small particles," Phys. Rev., vol. 70, no. 11-12, pp. 965-971, Dec. 1946.

[15] J. Frenkel and J. Doefman, "Spontaneous and Induced Magnetisation in Ferromagnetic Bodies," Nature, 
vol. 126, no. 3173, pp. 274-275, 1930.

[16] T. Mitsui and J. Furuichi, "Domain structure of rochelle salt and KH2PO4," Phys. Rev., vol. 90, no. 2, pp. 193-202, Apr. 1953.

[17] G. Catalan, J. F. Scott, A. Schilling, and J. M. Gregg, "Wall thickness dependence of the scaling law for ferroic stripe domains," J. Phys. Condens. Matter, vol. 19, no. 2, p. 022201, Dec. 2007.

[18] G. Catalan et al., "Fractal Dimension and Size Scaling of Domains in Thin Films of Multiferroic $\mathrm{BiFeO}_{3}$," Phys. Rev. Lett., vol. 100, no. 2, p. 027602, Jan. 2008.

[19] J. F. Scott, "Nanoferroelectrics: statics and dynamics.," J. Phys. Condens. Matter, vol. 18, no. 17, pp. R361-86, May 2006.

[20] M. Kläui, "Head-to-head domain walls in magnetic nanostructures," J. Phys. Condens. Matter, vol. 20, no. 31, p. 313001, 2008.

[21] E. M. Eyring, “Topics in Applied Physics," Nucl. Technol., vol. 39, no. 3, pp. 327-328, 1978.

[22] M. E. Holtz et al., "Topological Defects in Hexagonal Manganites: Inner Structure and Emergent Electrostatics," Nano Lett., vol. 17, no. 10, pp. 5883-5890, Oct. 2017.

[23] S. Choudhury et al., "The influence of $180^{\circ}$ ferroelectric domain wall width on the threshold field for wall motion," J. Appl. Phys., vol. 104, no. 8, p. 84107, Oct. 2008.

[24] J. F. Scott, D. M. Evans, J. M. Gregg, and A. Gruverman, "Hydrodynamics of domain walls in ferroelectrics and multiferroics: Impact on memory devices,” Appl. Phys. Lett., vol. 109, no. 4, p. 42901, Jul. 2016.

[25] F. Tsai, V. Khiznichenko, and J. M. Cowley, "High-resolution electron microscopy of $90^{\circ}$ ferroelectric domain boundaries in $\mathrm{BaTiO}_{3}$ and $\mathrm{Pb}\left(\mathrm{Zr}_{0.52} \mathrm{Ti}_{0.48}\right) \mathrm{O}_{3}$," Ultramicroscopy, vol. 45, no. 1, pp. 55-63, 1992.

[26] M. D. Dennis and R. C. Bradt, "Thickness of $90^{\circ}$ ferroelectric domain walls in (Ba,Pb)TiO3 single crystals," J. Appl. Phys., vol. 45, no. 5, pp. 1931-1933, May 1974.

[27] D. Lee et al., "Mixed Bloch-Néel-Ising character of $180^{\circ}$ ferroelectric domain walls," Phys. Rev. B Condens. Matter Mater. Phys., vol. 80, no. 6, pp. 2-5, 2009.

[28] L. Goncalves-Ferreira, S. A. T. Redfern, E. Artacho, and E. K. H. Salje, "Ferrielectric twin walls in CaTiO3," Phys. Rev. Lett., vol. 101, no. 9, p. 97602, Aug. 2008.

[29] A. K. Tagantsev, E. Courtens, and L. Arzel, "Prediction of a low-temperature ferroelectric instability in antiphase domain boundaries of strontium titanate," Phys. Rev. B - Condens. Matter Mater. Phys., vol. 64, no. 22, pp. 2241071-22410714, Nov. 2001.

[30] J. M. Gregg, "Exotic domain states in ferroelectrics: Searching for vortices and skyrmions," Ferroelectrics, vol. 433, no. 1, pp. 74-87, Jan. 2012.

[31] A. N. Morozovska, "Domain Wall Conduction in Ferroelectrics," Ferroelectrics, vol. 438, no. 1, pp. 319, Jan. 2012.

[32] V. Stepkova, P. Marton, and J. Hlinka, "Stress-induced phase transition in ferroelectric domain walls of BaTiO 3," J. Phys. Condens. Matter, vol. 24, no. 21, p. 212201, 2012.

[33] G. De Luca, M. D. Rossell, J. Schaab, N. Viart, M. Fiebig, and M. Trassin, "Domain Wall Architecture in Tetragonal Ferroelectric Thin Films," Adv. Mater., vol. 29, no. 7, p. 1605145, Feb. 2017.

[34] X.-K. Wei, C.-L. Jia, T. Sluka, B.-X. Wang, Z.-G. Ye, and N. Setter, "Néel-like domain walls in ferroelectric $\mathrm{Pb}(\mathrm{Zr}, \mathrm{Ti}) \mathrm{O}_{3}$ single crystals," Nat. Commun., vol. 7, p. 12385, Aug. 2016.

[35] S. Cherifi-Hertel et al., "Non-Ising and chiral ferroelectric domain walls revealed by nonlinear optical microscopy," Nat. Commun., vol. 8, p. 15768, Jun. 2017.

[36] N. Leo et al., "Polarization control at spin-driven ferroelectric domain walls," Nat. Commun., vol. 6, pp. 4-9, 2015.

[37] D. Meier et al., "Topology and manipulation of multiferroic hybrid domains in MnWO4," Phys. Rev. B Condens. Matter Mater. Phys., vol. 80, no. 22, p. 224420 , Dec. 2009.

[38] N. Domingo, S. Farokhipoor, J. Santiso, B. Noheda, and G. Catalan, "Domain wall magnetoresistance in BiFeO3 thin films measured by scanning probe microscopy," J. Phys. Condens. Matter, vol. 29, no. 33, p. 334003, Jul. 2017.

[39] M. Matsubara et al., "Magnetoelectric domain control in multiferroic TbMnO 3," Science (80-. )., vol. 348, no. 6239, pp. 1112-1115, Jun. 2015.

[40] S. A. Redfern and M. A. Carpenter, "Transformation Processes in Minerals.” De Gruyter, Berlin, Boston, 2018.

[41] M. E. Lines and A. M. Glass, Principles and Applications of Ferroelectrics and Related Materials. Oxford: Oxford University Press, 2010.

[42] E. K. H. Salje and M. A. Carpenter, "Linear - Quadratic order parameter coupling and multiferroic phase transitions," J. Phys. Condens. Matter, vol. 23, no. 46, p. 462202, 2011.

[43] E. K. Salje, Phase Transitions in Ferroelastic and Co-elastic Crystals. Cambridge University Press, 1991.

[44] M. A. Carpenter and E. K. H. Salje, "Elastic anomalies in minerals due to structural phase transitions," 
Eur. J. Mineral., vol. 10, no. 4, pp. 693-812, 1998.

[45] M. Fiebig, "Revival of the magnetoelectric effect," J. Phys. D. Appl. Phys., vol. 38, no. 8, pp. R123-R152, 2005 .

[46] T. Kimura, "Spiral Magnets as Magnetoelectrics," Annu. Rev. Mater. Res., vol. 37, no. 1, pp. 387-413, Aug. 2007.

[47] M. Mostovoy, "Ferroelectricity in Spiral Magnets," Phys. Rev. Lett., vol. 96, no. 6, p. 67601, Feb. 2006.

[48] T. Kimura, T. Goto, H. Shintani, K. Ishizaka, T. Arima, and Y. Tokura, "Magnetic control of ferroelectric polarization.," Nature, vol. 426, no. 6962, pp. 55-8, Nov. 2003.

[49] F. Kagawa et al., "Dynamics of multiferroic domain wall in spin-cycloidal ferroelectric DyMnO3," Phys. Rev. Lett., vol. 102, no. 5, p. 57604, Feb. 2009.

[50] B. Lorenz, “ Hexagonal Manganites-(RMnO 3 ): Class (I) Multiferroics with Strong Coupling of Magnetism and Ferroelectricity ," ISRN Condens. Matter Phys., vol. 2013, no. 1, pp. 1-43, 2013.

[51] W. Wu, Y. Geng, C. Fennie, M. Mostovoy, and S. Cheong, "Visualizing domain wall magnetism and domain magnetoelectricity in multiferroic hexagonal manganites," in 2015 IEEE International Magnetics Conference (INTERMAG), 2015, pp. 1-1.

[52] Y. Kumagai and N. A. Spaldin, "Structural domain walls in polar hexagonal manganites," Nat. Commun., vol. 4, p. 1540, Feb. 2013.

[53] D. Meier et al., "Anisotropic conductance at improper ferroelectric domain walls.," Nat. Mater., vol. 11, no. 4, pp. 284-8, Apr. 2012.

[54] D. R. Småbråten, Q. N. Meier, S. H. Skjærvø, K. Inzani, D. Meier, and S. M. Selbach, "Charged domain walls in improper ferroelectric hexagonal manganites and gallates," Phys. Rev. Mater., vol. 2, no. 11, p. 114405, Nov. 2018.

[55] W. Wu, Y. Horibe, N. Lee, S.-W. Cheong, and J. R. Guest, "Conduction of Topologically Protected Charged Ferroelectric Domain Walls,” Phys. Rev. Lett., vol. 108, no. 7, p. 77203, Feb. 2012.

[56] A. B. Mosberg et al., "FIB lift-out of conducting ferroelectric domain walls in hexagonal manganites," Appl. Phys. Lett., vol. 115, no. 12, p. 122901, Sep. 2019.

[57] T. Choi, Y. Horibe, H. T. Yi, Y. J. Choi, W. Wu, and S. W. Cheong, "Insulating interlocked ferroelectric and structural antiphase domain walls in multiferroic YMnO 3," Nat. Mater., vol. 9, no. 3, pp. 253-258, Mar. 2010.

[58] P. W. Turner et al., "Large Carrier Mobilities in ErMnO 3 Conducting Domain Walls Revealed by Quantitative Hall-Effect Measurements," Nano Lett., vol. 18, no. 10, pp. 6381-6386, Oct. 2018.

[59] J. A. Mundy et al., "Functional electronic inversion layers at ferroelectric domain walls," Nat. Mater., vol. 16, no. 6, p. 622, Mar. 2017.

[60] G. Catalan, J. Seidel, R. Ramesh, and J. F. Scott, "Domain wall nanoelectronics," Rev. Mod. Phys., vol. 84, no. 1, pp. 119-156, Feb. 2012.

[61] P. Sharma et al., "Nonvolatile ferroelectric domain wall memory," Sci. Adv., vol. 3, no. 6, 2017.

[62] J. R. Whyte et al., "Ferroelectric Domain Wall Injection,” Adv. Mater., vol. 26, no. 2, pp. 293-298, 2014.

[63] J. R. Whyte and J. M. Gregg, "A diode for ferroelectric domain-wall motion," Nat. Commun., vol. 6, p. 7361, Jun. 2015.

[64] P. S. Bednyakov, T. Sluka, A. K. Tagantsev, D. Damjanovic, and N. Setter, "Formation of charged ferroelectric domain walls with controlled periodicity,” Sci. Rep., vol. 5, p. 15819, Oct. 2015.

[65] D. A. Sanchez et al., "Room-temperature single phase multiferroic magnetoelectrics: $\mathrm{Pb}(\mathrm{Fe}, \mathrm{M})$ x(Zr,Ti)(1-x)O3 [M = Ta, Nb]," J. Appl. Phys., vol. 113, no. 7, p. 74105, Feb. 2013.

[66] P. S. Bednyakov, B. I. Sturman, T. Sluka, A. K. Tagantsev, and P. V Yudin, "Physics and applications of charged domain walls," npj Comput. Mater., vol. 4, no. 1, p. 65, 2018.

[67] M. Fiebig, T. Lottermoser, D. Meier, and M. Trassin, "The evolution of multiferroics," Nat. Rev. Mater., vol. 1, p. 16046, Aug. 2016.

[68] N. A. Spaldin and R. Ramesh, “Advances in magnetoelectric multiferroics," Nat. Mater., vol. 18, no. 3, pp. 203-212, 2019.

[69] S. Dong, H. Xiang, and E. Dagotto, "Magnetoelectricity in multiferroics: A theoretical perspective," Natl. Sci. Rev., vol. 6, no. 4, pp. 629-641, 2019.

[70] M. Campanini, R. Erni, and M. D. Rossell, "Probing local order in multiferroics by transmission electron microscopy,” Physical Sciences Reviews, vol. 0. pp. 1-36, 2019.

[71] W. J. Merz, "The electric and optical behavior of BaTiO3 single-domain crystals," Phys. Rev., vol. 76, no. 8, pp. 1221-1225, Oct. 1949.

[72] P. W. Forsbergh, "Domain Structures and Phase Transitions in Barium Titanate," Phys. Rev., vol. 76, no. 8, pp. 1187-1201, Oct. 1949.

[73] B. Matthias and A. von Hippel, "Domain Structure and Dielectric Response of Barium Titanate Single Crystals,” Phys. Rev., vol. 73, no. 11, pp. 1378-1384, Jun. 1948. 
[74] J. A. Hooton and W. J. Merz, "Etch patterns and ferroelectric domains in BaTiO3 single crystals," Phys. Rev., vol. 98, no. 2, pp. 409-413, Apr. 1955.

[75] T. H. E. Lahtinen, K. J. A. Franke, and S. van Dijken, "Electric-field control of magnetic domain wall motion and local magnetization reversal," Sci. Rep., vol. 2, no. 1, p. 258, Dec. 2012.

[76] M. Trassin et al., "Interfacial coupling in multiferroic/ferromagnet heterostructures," Phys. Rev. B Condens. Matter Mater. Phys., vol. 87, no. 13, p. 134426, Apr. 2013.

[77] Z. Zhou et al., "Probing electric field control of magnetism using ferromagnetic resonance," Nat. Commun., vol. 6, no. 1, p. 6082, Dec. 2015.

[78] I. Gross et al., "Real-space imaging of non-collinear antiferromagnetic order with a single-spin magnetometer," Nature, vol. 549, pp. 252-256, Sep. 2017.

[79] J. Y. Chauleau, E. Haltz, C. Carrétéro, S. Fusil, and M. Viret, "Multi-stimuli manipulation of antiferromagnetic domains assessed by second-harmonic imaging," Nat. Mater., vol. 16, no. 8, pp. 803807, May 2017.

[80] A. Gruverman, M. Alexe, and D. Meier, "Piezoresponse force microscopy and nanoferroic phenomena," Nat. Commun., vol. 10, no. 1, p. 1661, 2019.

[81] L. Rondin, J. P. Tetienne, T. Hingant, J. F. Roch, P. Maletinsky, and V. Jacques, "Magnetometry with nitrogen-vacancy defects in diamond," Reports Prog. Phys., vol. 77, no. 5, p. 056503, May 2014.

[82] D. Lebeugle, D. Colson, A. Forget, M. Viret, A. M. Bataille, and A. Gukasov, "Electric-Field-Induced Spin Flop in $\mathrm{BiFeO}_{3}$ Single Crystals at Room Temperature," Phys. Rev. Lett., vol. 100, no. 22, p. 227602, Jun. 2008.

[83] D. Sando et al., "Crafting the magnonic and spintronic response of BiFeO 3 films by epitaxial strain," Nat. Mater., vol. 12, no. 7, pp. 641-646, Jul. 2013.

[84] I. Sosnowska, T. P. Neumaier, and E. Steichele, "Spiral magnetic ordering in bismuth ferrite," J. Phys. C Solid State Phys., vol. 15, no. 23, pp. 4835-4846, 1982.

[85] T. Zhao et al., "Electrical control of antiferromagnetic domains in multiferroic BiFeO 3 films at room temperature," Nat. Mater., vol. 5, no. 10, pp. 823-829, Oct. 2006.

[86] M. Fiebig, T. Lottermoser, D. Fröhlich, A. V. Goltsev, and R. V. Pisarev, "Observation of coupled magnetic and electric domains," Nature, vol. 419, no. 6909, pp. 818-820, Oct. 2002.

[87] M. Fiebig et al., "Determination of the magnetic symmetry of hexagonal manganites by second harmonic generation," Phys. Rev. Lett., vol. 84, no. 24, pp. 5620-5623, Jun. 2000.

[88] J. Nordlander, G. De Luca, N. Strkalj, M. Fiebig, and M. Trassin, "Probing ferroic states in oxide thin films using optical second harmonic generation," Appl. Sci., vol. 8, no. 4, 2018.

[89] S. Valencia et al., "Interface-induced room-temperature multiferroicity in $\mathrm{BaTiO}_{3}$," Nat. Mater., vol. 10, p. 753, Aug. 2011.

[90] J. Seidel et al., "Domain wall conductivity in La-doped BiFeO 3," Phys. Rev. Lett., vol. 105, no. 19, p. 197603, Nov. 2010.

[91] E. K. H. Salje and J. C. Lashley, "Domain boundary engineering in ferroic and multiferroic materials: A simple introduction," in Springer Series in Materials Science, vol. 148, no. 1, T. Kakeshita, T. Fukuda, A. Saxena, and A. Planes, Eds. Berlin, Heidelberg: Springer Berlin Heidelberg, 2011, pp. 1-18.

[92] J. F. Scott, "Room-temperature multiferroic magnetoelectrics," Npg Asia Mater., vol. 5, p. e72, Nov. 2013.

[93] D. Meier, M. Maringer, T. Lottermoser, P. Becker, L. Bohatý, and M. Fiebig, "Observation and coupling of domains in a spin-spiral multiferroic," Phys. Rev. Lett., vol. 102, no. 10, p. 107202, Mar. 2009.

[94] J. Seidel et al., "Conduction at domain walls in oxide multiferroics," Nat. Mater., vol. 8, no. 3, pp. 229234, Mar. 2009.

[95] J. Padilla, W. Zhong, and D. Vanderbilt, "First-principles investigation of $180^{\circ}$ domain walls in BaTO3," Phys. Rev. B - Condens. Matter Mater. Phys., vol. 53, no. 10, pp. R5969-R5973, Mar. 1996.

[96] R. D. McMichael and M. J. Donahue, "Head to head domain wall structures in thin magnetic strips," IEEE Trans. Magn., vol. 33, no. 5 PART 2, pp. 4167-4169, 1997.

[97] A. G. G. Chynoweth and W. L. L. Feldmann, "Ferroelectric domain delineation in triglycine sulphate and domain arrays produced by thermal shocks," J. Phys. Chem. Solids, vol. 15, no. 3, pp. 225-233, Oct. 1960.

[98] A. A. Grekov, A. A. Adonin, and N. P. Protsenko, "Encountering domains in sbsi," Ferroelectrics, vol. 13, no. 1, pp. 483-485, Jan. 1976.

[99] E. G. Fesenko, V. G. Gavrilyatchenko, M. A. Martinenko, A. F. Semenchov, and I. P. Lapin, "Domain structure peculiarities of lead-titanate crystals," Ferroelectrics, vol. 6, no. 1, pp. 61-65, Jan. 1973.

[100] Z. Surowiak, J. Dec, R. Skulski, E. G. Fesenko, V. G. Gavrilyatchenko, and A. F. Semenchev, "The domain structure formation at phase transitions," Ferroelectrics, vol. 20, no. 1, pp. 277-279, Jan. 1978.

[101] F. Jona and G. Shirane, Ferroelectric Crystals, vol. 43, no. 10-11. John Wiley \& Sons, Ltd, 1963.

[102] V. V. Aristov, L. S. Kokhanchik, K. -P Meyer, and H. Blumtritt, "Scanning electron microscopic investigations of peculiarities of the BaTiO3 ferroelectric domain contrast," Phys. Status Solidi, vol. 78, 
no. 1, pp. 229-236, Jul. 1983.

[103] V. V Aristov, L. S. Kokhanchik, and Y. I. Voronovskii, "Voltage contrast of ferroelectric domains of lithium niobate in SEM," Phys. status solidi, vol. 86, no. 1, pp. 133-141, Nov. 1984.

[104] A. A. Sogr, "Domain structure of ferroelectrics observed in the scanning electron microscope," Ferroelectrics, vol. 97, no. 1, pp. 47-57, Sep. 1989.

[105] J. Guyonnet, I. Gaponenko, S. Gariglio, and P. Paruch, "Conduction at domain walls in insulating $\mathrm{Pb}(\mathrm{Zr}$ 0.2Ti 0.8)O 3 thin films,” Adv. Mater., vol. 23, no. 45, pp. 5377-5382, Dec. 2011.

[106] A. Tselev et al., "Microwave a.c. conductivity of domain walls in ferroelectric thin films," Nat. Commun., vol. 7, no. May, p. 11630, May 2016.

[107] I. Stolichnov et al., "Bent Ferroelectric Domain Walls as Reconfigurable Metallic-Like Channels," Nano Lett., vol. 15, no. 12, pp. 8049-8055, Dec. 2015.

[108] M. Schröder, A. Haußmann, A. Thiessen, E. Soergel, T. Woike, and L. M. Eng, "Conducting Domain Walls in Lithium Niobate Single Crystals," Adv. Funct. Mater., vol. 22, no. 18, pp. 3936-3944, Sep. 2012.

[109] C. Godau, T. Kämpfe, A. Thiessen, L. M. Eng, and A. Haußmann, "Enhancing the Domain Wall Conductivity in Lithium Niobate Single Crystals," ACS Nano, vol. 11, no. 5, pp. 4816-4824, May 2017.

[110] C. S. Werner et al., "Large and accessible conductivity of charged domain walls in lithium niobate," Sci. Rep., vol. 7, no. 1, p. 9862, 2017.

[111] T. Sluka, A. K. Tagantsev, P. Bednyakov, and N. Setter, "Free-electron gas at charged domain walls in insulating BaTiO3,” Nat. Commun., vol. 4, no. May, p. 1808, Jan. 2013.

[112] M. P. Campbell, J. P. V. V McConville, R. G. P. P. McQuaid, D. Prabhakaran, A. Kumar, and J. M. Gregg, "Hall effect in charged conducting ferroelectric domain walls," Nat. Commun., vol. 7, p. 13764, Dec. 2016.

[113] J. Schaab et al., "Electrical half-wave rectification at ferroelectric domain walls," Nat. Nanotechnol., vol. 13, no. 11, pp. 1028-1034, 2018.

[114] J. Schaab et al., "Imaging and characterization of conducting ferroelectric domain walls by photoemission electron microscopy," Appl. Phys. Lett., vol. 104, no. 23, p. 232904 , Jun. 2014.

[115] J. Nordlander et al., "The ultrathin limit of improper ferroelectricity," Nat. Commun., vol. 10, no. 1, p. 5591, 2019.

[116] R. G. P. McQuaid, M. P. Campbell, R. W. Whatmore, A. Kumar, and J. M. Gregg, "Injection and controlled motion of conducting domain walls in improper ferroelectric $\mathrm{Cu}-\mathrm{Cl}$ boracite," Nat. Commun., vol. 8, p. 15105, May 2017.

[117] Y. S. Oh, X. Luo, F. T. Huang, Y. Wang, and S. W. Cheong, "Experimental demonstration of hybrid improper ferroelectricity and the presence of abundant charged walls in (Ca,Sr)3Ti2O7 crystals," Nat. Mater., vol. 14, no. 4, pp. 407-413, Jan. 2015.

[118] J. Jiang et al., "Temporary formation of highly conducting domain walls for non-destructive read-out of ferroelectric domain-wall resistance switching memories," Nat. Mater., vol. 17, no. 1, pp. 49-55, Nov. 2018.

[119] A. Q. Jiang and Y. Zhang, "Next-generation ferroelectric domain-wall memories: principle and architecture," NPG Asia Mater., vol. 11, no. 1, p. 2, 2019.

[120] A. Crassous, T. Sluka, A. K. Tagantsev, and N. Setter, "Polarization charge as a reconfigurable quasidopant in ferroelectric thin films," Nat. Nanotechnol., vol. 10, no. 7, pp. 614-618, 2015.

[121] D. Meier, "Functional domain walls in multiferroics," J. Phys. Condens. Matter, vol. 27, no. 46, 2015.

[122] S. Farokhipoor and B. Noheda, "Conduction through $71^{\circ}$ domain walls in BiFeO3 thin films," Phys. Rev. Lett., vol. 107, no. 12, pp. 1-4, Apr. 2011.

[123] T. Jungk, Á. Hoffmann, M. Fiebig, and E. Soergel, "Electrostatic topology of ferroelectric domains in $\mathrm{YMnO}_{3}$," Appl. Phys. Lett., vol. 97, no. 1, p. 012904, Jul. 2010.

[124] P. Schoenherr et al., "Observation of Uncompensated Bound Charges at Improper Ferroelectric Domain Walls," Nano Lett., vol. 19, no. 3, pp. 1659-1664, Mar. 2019.

[125] S. H. Skjærvø, D. R. Småbråten, N. A. Spaldin, T. Tybell, and S. M. Selbach, "Oxygen vacancies in the bulk and at neutral domain walls in hexagonal YMnO3," Phys. Rev. B, vol. 98, no. 18, p. 184102, Nov. 2018.

[126] S. H. Skjærvø et al., "Interstitial oxygen as a source of p-type conductivity in hexagonal manganites," Nat. Commun., vol. 7, p. 13745, Dec. 2016.

[127] X. Wang, F. T. Huang, R. Hu, F. Fan, and S. W. Cheong, "Self-poling with oxygen off-stoichiometry in ferroelectric hexagonal manganites," APL Mater., vol. 3, no. 4, p. 41505, Feb. 2015.

[128] S. M. Selbach, A. Nordli Løvik, K. Bergum, J. R. Tolchard, M.-A. Einarsrud, and T. Grande, "Crystal structure, chemical expansion and phase stability of $\mathrm{HoMnO}_{3}$ at high temperature," J. Solid State Chem., vol. 196, pp. 528-535, 2012.

[129] G. J. McCarthy, P. V. Gallagher, and C. Sipe, "Crystal chemistry of catalyst materials. I. Composition and unit cell parameters of 'REMnO3' phases prepared in air," Mater. Res. Bull., vol. 8, no. 11, pp. 1277- 
$1284,1973$.

[130] S. M. Griffin, M. Reidulff, S. M. Selbach, and N. A. Spaldin, "Defect Chemistry as a Crystal Structure Design Parameter: Intrinsic Point Defects and Ga Substitution in $\mathrm{InMnO}_{3}$," Chem. Mater., vol. 29, no. 6, pp. 2425-2434, Mar. 2017.

[131] T. S. Holstad et al., "Electronic bulk and domain wall properties in B -site doped hexagonal ErMnO3," Phys. Rev. B, vol. 97, no. 8, p. 85143, Feb. 2018.

[132] E. Hassanpour et al., "Robustness of magnetic and electric domains against charge carrier doping in multiferroic hexagonal ErMnO3," New J. Phys., vol. 18, no. 4, p. 43015, 2016.

[133] Q. N. Meier et al., "Global formation of topological defects in the multiferroic hexagonal manganites," Phys. Rev. X, vol. 7, no. 4, p. 41014, Oct. 2017.

[134] S. M. Griffin, M. Lilienblum, K. T. Delaney, Y. Kumagai, M. Fiebig, and N. A. Spaldin, "Scaling behavior and beyond equilibrium in the hexagonal manganites," Phys. Rev. X, vol. 2, no. 4, p. 41022, Dec. 2012.

[135] S. M. Griffin and N. A. Spaldin, "On the relationship between topological and geometric defects," J. Phys. Condens. Matter, vol. 29, no. 34, p. 343001, 2017.

[136] N. Balke et al., "Deterministic control of ferroelastic switching in multiferroic materials," Nat. Nanotechnol., vol. 4, no. 12, pp. 868-875, Dec. 2009.

[137] A. Lubk, S. Gemming, and N. A. Spaldin, "First-principles study of ferroelectric domain walls in multiferroic bismuth ferrite," Phys. Rev. B, vol. 80, no. 10, p. 104110, Sep. 2009.

[138] M. Daraktchiev, G. Catalan, and J. F. Scott, "Landau theory of domain wall magnetoelectricity," Phys. Rev. B - Condens. Matter Mater. Phys., vol. 81, no. 22, p. 224118, Jun. 2010.

[139] M. R. Scheinfein, J. Unguris, M. H. Kelley, D. T. Pierce, and R. J. Celotta, "Scanning electron microscopy with polarization analysis (SEMPA)," Rev. Sci. Instrum., vol. 61, no. 10, pp. 2501-2527, Oct. 1990.

[140] J. X. Zhang, R. J. Zeches, Q. He, Y. H. Chu, and R. Ramesh, "Nanoscale phase boundaries: A new twist to novel functionalities," Nanoscale, vol. 4, no. 20, pp. 6196-6204, 2012.

[141] Q. He et al., "Magnetotransport at domain walls in BiFeO 3," Phys. Rev. Lett., vol. 108, no. 6, pp. 1-5, 2012.

[142] Y. Geng, N. Lee, Y. J. Choi, S. W. Cheong, and W. Wu, "Collective magnetism at multiferroic vortex domain walls," Nano Lett., vol. 12, no. 12, pp. 6055-6059, 2012.

[143] D. Meier et al., "Mutual induction of magnetic 3d and 4f order in multiferroic hexagonal ErMnO 3," Phys. Rev. B - Condens. Matter Mater. Phys., vol. 86, no. 18, p. 184415, Nov. 2012.

[144] S. Farokhipoor et al., "Artificial chemical and magnetic structure at the domain walls of an epitaxial oxide," Nature, vol. 515, no. 7527, pp. 379-383, 2014.

[145] D. M. Juraschek, M. Fechner, A. V. Balatsky, and N. A. Spaldin, “Dynamical multiferroicity,” Phys. Rev. Mater., vol. 1, no. 1, p. 14401, Jun. 2017.

[146] J. T. Heron et al., "Electric-field-induced magnetization reversal in a ferromagnet-multiferroic heterostructure," Phys. Rev. Lett., vol. 107, no. 21, p. 217202, Nov. 2011.

[147] M. Bibes and A. Barthélémy, "Multiferroics: Towards a magnetoelectric memory," Nat. Mater., vol. 7, no. 6, pp. 425-426, 2008.

[148] J. T. Heron et al., "Deterministic switching of ferromagnetism at room temperature using an electric field.," Nature, vol. 516, no. 7531, pp. 370-3, Dec. 2014.

[149] C. Ederer and N. A. Spaldin, "Influence of strain and oxygen vacancies on the magnetoelectric properties of multiferroic bismuth ferrite," Phys. Rev. B - Condens. Matter Mater. Phys., vol. 71, no. 22, p. 224103, Jun. 2005.

[150] P. Rovillain et al., "Electric-field control of spin waves at room temperature in multiferroic $\mathrm{BiFeO}_{3}$," $\mathrm{Nat}$. Mater., vol. 9, no. 12, pp. 975-979, Dec. 2010.

[151] T. Jungwirth, X. Marti, P. Wadley, and J. Wunderlich, "Antiferromagnetic spintronics," Nat. Nanotechnol., vol. 11, no. 3, pp. 231-241, 2016.

[152] J. F. Scott, D. M. Evans, R. S. Katiyar, R. G. P. McQuaid, and J. M. Gregg, "Nonequilibrium ferroelectricferroelastic $10 \mathrm{~nm}$ nanodomains: wrinkles, period-doubling, and power-law relaxation," J. Phys. Condens. Matter, vol. 29, no. 30, p. 304001, Jun. 2017.

[153] M. D. Glinchuk, E. A. Eliseev, and A. N. Morozovska, "Novel room temperature multiferroics on the base of single-phase nanostructured perovskites," J. Appl. Phys., vol. 116, no. 5, p. 54101, Aug. 2014.

[154] M. D. Glinchuk, E. A. Eliseev, and A. N. Morozovska, "Landau-Ginzburg description of anomalous properties of novel room temperature multiferroics $\mathrm{Pb}(\mathrm{Fe} 1 / 2 \mathrm{Ta} 1 / 2) \mathrm{x}(\mathrm{Zr} 0.53 \mathrm{Ti} 0.47) 1-\mathrm{xO} 3$ and Pb(Fe1/2Nb1/2)x(Zr0.53Ti0.47)1-xO3," J. Appl. Phys., vol. 119, no. 2, p. 24102, Jan. 2016.

[155] Y.-C. Wu et al., "Room Temperature Multiferroic PZTFT Thin Films," ACS Appl. Electron. Mater., Dec. 2019.

[156] D. M. Evans et al., "Magnetic switching of ferroelectric domains at room temperature in multiferroic PZTFT," Nat. Commun., vol. 4, p. 1534, Feb. 2013. 
[157] D. M. Evans et al., "Switching ferroelectric domain configurations using both electric and magnetic fields in $\mathrm{Pb}(\mathrm{Zr}, \mathrm{Ti}) \mathrm{O} 3-\mathrm{Pb}(\mathrm{Fe}, \mathrm{Ta}) \mathrm{O} 3$ single-crystal lamellae," Philos. Trans. R. Soc. A Math. Phys. Eng. Sci., vol. 372, no. 2009, p. 20120450, Feb. 2014.

[158] J. Schiemer et al., "Studies of the room-temperature multiferroic $\mathrm{Pb}(\mathrm{Fe} 0.5 \mathrm{Ta} 0.5) 0.4(\mathrm{Zr} 0.53 \mathrm{Ti0} 0.47)$ 0.6O3: Resonant ultrasound spectroscopy, dielectric, and magnetic phenomena," Adv. Funct. Mater., vol. 24, no. 20, pp. 2993-3002, May 2014.

[159] D. M. Evans et al., "The Nature of Magnetoelectric Coupling in $\mathrm{Pb}(\mathrm{Zr}, \mathrm{Ti}) \mathrm{O} 3-\mathrm{Pb}(\mathrm{Fe}, \mathrm{Ta}) \mathrm{O} 3$, , Adv. Mater., vol. 27, no. 39, pp. 6068-6073, 2015.

[160] D. A. Sanchez et al., "Symmetries and multiferroic properties of novel room-temperature magnetoelectrics: Lead iron tantalate - lead zirconate titanate (PFT/PZT)," AIP Adv., vol. 1, no. 4, p. 42169, Dec. 2011.

[161] A. P. Pyatakov et al., "Magnetically switched electric polarity of domain walls in iron garnet films," Epl, vol. 93, no. 1, p. 17001, 2011.

[162] K. Taniguchi, N. Abe, T. Takenobu, Y. Iwasa, and T. Arima, "Ferroelectric polarization flop in a frustrated magnet MnWO4 induced by a magnetic field," Phys. Rev. Lett., vol. 97, no. 9, p. 097203, Aug. 2006.

[163] S. Manz et al., "Reversible optical switching of antiferromagnetism in TbMnO3," Nat. Photonics, vol. 10, no. 10, pp. 653-656, Aug. 2016.

[164] I. I. Naumov, L. Bellaiche, and H. Fu, "Unusual phase transitions in ferroelectric nanodisks and nanorods," Nature, vol. 432, no. 7018, pp. 737-740, Dec. 2004.

[165] A. Gruverman et al., "Vortex ferroelectric domains," J. Phys. Condens. Matter, vol. 20, no. 34, p. 342201, Aug. 2008.

[166] J. Ma et al., "Controllable conductive readout in self-assembled, topologically confined ferroelectric domain walls," Nat. Nanotechnol., vol. 13, no. 10, pp. 947-952, Oct. 2018.

[167] K. E. Kim et al., "Configurable topological textures in strain graded ferroelectric nanoplates," Nat. Commun., vol. 9, no. 1, pp. 1-12, 2018.

[168] Z. Li et al., "High-density array of ferroelectric nanodots with robust and reversibly switchable topological domain states,"Sci. Adv., vol. 3, no. 8, p. e1700919, Aug. 2017.

[169] N. Balke et al., "Enhanced electric conductivity at ferroelectric vortex cores in BiFeO 3," Nat. Phys., vol. 8, no. 1, pp. 81-88, 2012.

[170] M. A. P. Gonçalves, C. Escorihuela-Sayalero, P. Garca-Fernández, J. Junquera, and J. Íñiguez, "Theoretical guidelines to create and tune electric skyrmion bubbles," Sci. Adv., vol. 5, no. 2, p. eaau7023, Feb. 2019.

[171] Y. Nahas, S. Prokhorenko, L. Louis, Z. Gui, I. Kornev, and L. Bellaiche, "Discovery of stable skyrmionic state in ferroelectric nanocomposites," Nat. Commun., vol. 6, no. May, pp. 1-6, 2015.

[172] S. Das et al., "Observation of room-temperature polar skyrmions," Nature, vol. 568, no. 7752, pp. 368372, 2019.

[173] M. Hoffmann et al., "Topological orbital magnetization and emergent Hall effect of an atomic-scale spin lattice at a surface," Phys. Rev. B - Condens. Matter Mater. Phys., vol. 92, no. 2, p. 020401, Jul. 2015.

[174] J. Hanke, F. Freimuth, S. Blügel, and Y. Mokrousov, "Prototypical topological orbital ferromagnet $\gamma$ FeMn," Sci. Rep., vol. 7, no. 1, p. 41078, Dec. 2017.

[175] K. Taguchi and G. Tatara, "Inverse Faraday Effect Driven by Spin Chirality in Weak Ferromagnets," J. Korean Phys. Soc., vol. 62, no. 12, pp. 1759-1762, 2013.

[176] R. K. Vasudevan et al., "Domain wall conduction and polarization-mediated transport in ferroelectrics," Adv. Funct. Mater., vol. 23, no. 20, pp. 2592-2616, May 2013.

[177] R. Lebrun et al., "Tunable long-distance spin transport in a crystalline antiferromagnetic iron oxide," Nature, vol. 561, no. 7722, pp. 222-225, 2018.

[178] F.-T. Huang and S.-W. Cheong, "Aperiodic topological order in the domain configurations of functional materials," Nat. Rev. Mater., vol. 2, no. 3, p. 17004, 2017. 